\title{
Stabilization of cycles with stochastic prediction-based and target-oriented control
}

E. Braverman, ${ }^{1}$ C. Kelly, ${ }^{2}$ and A. Rodkina ${ }^{3}$

${ }^{1)}$ Department of Mathematics and Statistics, University of Calgary,

2500 University Drive N.W., Calgary, AB T2N 1N4, Canadda)

2) School of Mathematical Sciences, University College Cork, Western Road, T12YT20, Cork, Ireland

${ }^{3)}$ Department of Mathematics, The University of the West Indies, Mona Campus, Kingston 7, Jamaictc)

(Dated: 22 December 2020)

We stabilize a prescribed cycle or an equilibrium of a difference equation using pulsed stochastic control. Our technique, inspired by Kolmogorov's Law of Large Numbers, activates a stabilizing effect of stochastic perturbation and allows for stabilization using a much wider range for the control parameter than would be possible in the absence of noise.

Our main general result applies to both Prediction-Based and Target-Oriented Controls. This analysis is the first to make use of the stabilizing effects of noise for Prediction-Based Control; the stochastic version has previously been examined in the literature, but only the destabilizing effect of noise was demonstrated. A stochastic variant of Target-Oriented Control has never been considered, to the best of our knowledge, and we propose a specific form that uses a point equilibrium or one point on a cycle as a target. We illustrate our results numerically on the logistic, Ricker and Maynard Smith models from population biology.

PACS numbers: 05.45.-a Nonlinear dynamics and chaos; 02.50.Fg Stochastic analysis; 05.45.Gg Control of chaos, applications of chaos

Keywords: stochastic difference equations; state dependent noise; stabilization of cycles; Prediction-Based Control, Target-Oriented Control; population models

Various linear-type methods were developed to control otherwise unstable or chaotic behaviour of discrete maps. Prediction-Based Control introduced by Ushio and Yamamoto in 1999 and twoparameter Target-Oriented Control proposed by Dattani et al in 2011 are among them. Stochastic perturbations were usually considered in two different contexts: as an intrinsic part of control which could diminish stabilization effects and thus should be kept in prescribed bounds, and as natural environmental noise which may somehow control chaos. For instance, such noise can reduce oscillation amplitudes.

First, we consider control types incorporating both deterministic and stochastic components, both of which can have a stabilizing effect. Examples illustrate that stabilization can be achieved by noise but introduction of deterministic control, which cannot stabilize in itself, can influence the bounds for stabilizing stochastic perturbations. We analyze how the effective range of stabilizing control parameters may be extended by the introduction of noise.

Second, stabilization of either an unstable equilibrium or an unstable orbit of a discrete equation

\footnotetext{
a) Electronic mail: maelena@ucalgary.ca

b) Electronic mail: conall.kelly@ucc.ie

c) Electronic mail: alexandra.rodkina@uwimona.edu.jm
}

is investigated. In addition, both regular (applied at each step) and pulsed (applied every $k$ th step) types of control are applied, and pulsed control can stabilize an equilibrium.

Third, stochastic control is considered in very general settings. These results are later applied to Target-Oriented and Prediction-Based types of control involving stochastic component in the control parameter. The application of stochastic Prediction-Based and Target-Oriented Controls for $k$-cycle stabilization is novel, stochastic versions of Target-Oriented Control have not been studied before.

\section{INTRODUCTION}

We investigate the use of pulsed stochastic control to stabilize a prescribed cycle of the difference equation

$$
x_{n+1}=f\left(x_{n}\right), \quad n \in \mathbb{N}_{0}, \quad x_{0}>0,
$$

where $\mathbb{N}_{0}:=\mathbb{N} \cup\{0\}$. For a general class of control methods applied to (11), we reduce this problem to the stabilization of a point equilibrium at zero and present a general theorem on pulsed stabilization of the zero equilibrium to equation (1).

We show how this theorem may be applied for two specific control methods: Prediction-Based Control (PBC) and a particular case of Target-Oriented Control (TOC). 
We introduce stochastic versions of both methods and study the interplay of the underlying control with stochastic perturbation of the control parameter, establishing results that show when the introduction of noise is beneficial for stabilization. In particular, we describe the stabilization of either a point equilibrium or a cycle by noise in the context of stochastic control. We also investigate the implications of pulsed control in this setting.

Our analysis allows us to demonstrate the stabilization of cycles for three commonly used models from population biology - Ricker, logistic and Maynard Smith - and we note that while stabilization of a point equilibrium by noise is quite a well-developed topic, stochastic stabilization of cycles is much less so.

Our technique is inspired by Kolmogorov's Law of Large Numbers which allows us to characterize the stabilizing effect of noise in our analysis, and may be stated as follows:

Lemma I.1 34 (page 391) Let $\left(v_{n}\right)_{n \in \mathbb{N}}$ be a sequence of independent identically distributed random variables where $\mathbb{E}\left|v_{n}\right|<\infty, n \in \mathbb{N}$. Denote the common mean $\mu:=\mathbb{E} v_{n}$, and the partial sum $S_{n}:=\sum_{k=1}^{n} v_{k}$. Then $\lim _{n \rightarrow \infty} S_{n} / n=\mu$, a.s.

To the best of our knowledge, the idea of stabilization by noise goes back to 1950s for physical applications. Consider the well-known pendulum of Kapica ${ }^{22}$, where stochastic perturbations can stabilize its top (otherwise unstable) position. This stabilizing effect is stipulated by the type of noise and its intensity: noise that is too intense does not lead to stabilization, but noise that is insufficiently intense leads only to the preservation of the stability of the bottom equilibrium. For differential equations, a theoretical justification of stabilization by noise originated in the 1960s; see Hasminskii 19 . For both differential and difference equations, more detailed historical notes, as well as recent results on the topic are given in $7,9,20$. Recently, stability and stabilization of stochastic difference equations and systems, as well as cyclic and chaotic behaviour, has become a focus of many publications $2,3,12,18,21,27,30,33$. Moreover, a developed theory of random difference equations was utilized to investigate differential equations $\frac{11}{11}$, or discrete and continuous stochastic equations were considered in the framework of a single model 14 .

$\mathrm{PBC}$ was first introduced by Ushio and Yamamoto 37 and was studied in detail in 25 . The case when the control is applied to (1) at every step, and the control parameter $\alpha \in(0,1)$ is subject to a stochastic perturbation, can be written

$x_{n+1}=f\left(x_{n}\right)-\left(\alpha+l \xi_{n+1}\right)\left(f\left(x_{n}\right)-x_{n}\right), n \in \mathbb{N}_{0}, x_{0}>0$,

and was considered in $\underline{7}^{7}$. If the control is applied at every $k$ th step, for $k \in \mathbb{N}, k>1$, then this is called pulsed control, and in the case of deterministic PBC it was investigated in $\underline{8,26}$.
Previous results on PBC view stabilization as arising from the deterministic control and in spite of the presence of stochastic perturbations of low intensity. However, we can show that it is possible to stabilize the equilibrium of (11) by stochastic PBC (2), even for values of $\alpha$ which do not deliver stability in the absence of noise.

TOC, applied to (11) at every step with target $T$, is characterized by

$$
x_{n+1}=f\left(\alpha T+(1-\alpha) x_{n}\right), \quad T \geq 0, \quad \alpha \in[0,1) .
$$

It was introduced in $\underline{13}$ and further investigated in $\underline{4}, \underline{15}$. $\mathrm{In}^{15}$ it was shown that TOC is topologically equivalent to the modified TOC equation

$$
x_{n+1}=\alpha T+(1-\alpha) f\left(x_{n}\right), \quad T \geq 0, \quad \alpha \in[0,1) .
$$

Note that in (2) and other stochastic control models with $f:[0, \infty) \rightarrow[0, \infty)$, once the control $\alpha+l \xi_{n+1} \in$ $[0,1]$, or, for TOC, the target is in addition non-negative, the expression in the right-hand side is non-negative. Assuming $\alpha \in[0,1),\left|\xi_{n+1}\right| \leq 1$, we get $l \leq \min \{\alpha, 1-\alpha\}$. However, most of our results are local, and we can consider parameters outside of this domain, considering the truncated version when the right-hand side is a maximum of the computed value and zero, which is quite a typical approach in population ecology 3 ㄹ.

The application of modified TOC to stabilize cycles in the context of higher order or vector difference equations was considered in $\underline{\underline{5} \underline{6}}$. In the present article we find a relationship between the control parameter $\alpha$ and the noise intensity $l$, which guarantees local stability of a cycle after application of stochastic pulsed stabilization. In the case when the control parameter is such that the unperturbed model is stable, our method provides conditions on the noise intensity which preserve stability, similarly to ${ }^{\underline{7}}$. In the case when a deterministic system is unstable after application of the control, introduction of a noise with appropriate intensity guarantees stability.

Pulsed control is essential in cases where application of control at each step is either impossible or inefficient from a practical or economical point of view. Here we consider linear types of control for nonlinear models with either one for PBC or two parameters for TOC involved. Our control results are robust as a result of the simplicity of the control structures and the continuity of the maps. While stabilization of an unstable equilibrium with a control applied at every step is always possible, once the control intensity is sufficient, pulsed stabilization with $\mathrm{PBC}$ in the deterministic case is problematio sometimes it cannot be achieved for any values of the parameters. In our earlier paper ${ }^{\underline{7}}$ a stochastic perturbation of deterministic PBC was explored but global stability was justified only in the case when all the values of the noisy control are within a range of parameters leading to stabilization in the deterministic case. In contrast with these global results, highly local results are obtained in ${ }^{9}$ where control and stabilization are achieved solely by noise but the neighbourhood of the equilibrium should be 
very small (sometimes less than $10^{-8}$ ) and thus anyway another control method is required in practical applications. Here our purpose is to combine a stabilizing effect of noise with PBC, expanding the range of allowable control parameters compared to $\underline{\underline{z}}$ and relaxing requirements on proximity of the initial value to the equilibrium in $\underline{9}$.

For TOC, delayed versions and stage-structed dynamics were considered in $\underline{\underline{4}} \underline{\underline{6}}$, but its stochastic version have never been explored. Here we obtain sufficient stabilization conditions for noisy TOC, including its pulsed version. And again, noise can expand the range of parameters for which stabilization is achieved. From this point of view, results of the present paper significantly generalize, for example $\stackrel{4}{\stackrel{4}{.}}$

All stochastic sequences considered in the paper are defined on a complete filtered probability space $(\Omega, \mathcal{F}$, $\left.\left\{\mathcal{F}_{n}\right\}_{n \in \mathbb{N}}, \mathbb{P}\right)$, where the filtration $\left(\mathcal{F}_{n}\right)_{n \in \mathbb{N}}$ is naturally generated by the sequence $\left(\xi_{n}\right)_{n \in \mathbb{N}}$, so that $\mathcal{F}_{n}=$ $\sigma\left\{\xi_{1}, \ldots, \xi_{n}\right\}$. We use the standard abbreviations "a.s." for either "almost sure" or "almost surely" with respect to a fixed probability measure $\mathbb{P}$, and "i.i.d." for "independent and identically distributed", as it applies to sequences of random variables. A detailed discussion of relevant stochastic concepts and notation can be found, for example, in ${ }^{34}$.

Since equations in the present paper are motivated by population models, we will assume bounded stochastic perturbations in the following sense:

Assumption I.2 $\left(\xi_{n}\right)_{n \in \mathbb{N}}$ is a sequence of independent identically distributed random variables, each satisfying $\left|\xi_{n}\right| \leq 1$.

The paper is organized as follows. The main stabilization theorem is presented in Section III in its most general form. A stochastic TOC method is introduced and discussed in Section IIA Results obtained in Section IIB for stochastic PBC are generalizations of $\mathbf{T}^{7}$. In Section [II] we illustrate some of our results with computer simulations. Section IV contains a brief summary and discussion of potential directions for future research. All proofs are deferred to an Appendix in Section IVB.

\section{LOCAL STABILIZATION OF A POINT EQUILIBRIUM AT ZERO BY PULSED STOCHASTIC CONTROL}

In this section we present a generalized control theorem that will be applied to specific classes of model and control-type in the remainder of the article. Consider the difference equation

$$
z_{n+1}=g\left(z_{n}\right), \quad n \in \mathbb{N}_{0}, \quad z_{0}>0,
$$

where the function $g$ satisfies a Lipschitz-type condition locally around zero:
Assumption II.1 For some $u_{0}>0$, there exists $L \geq 1$ such that

$$
|g(z)| \leq L|z|, \quad|z| \leq u_{0} .
$$

Condition (6) in Assumption II.1 is sufficient to ensure that equation (5) has a point equilibrium at zero, which we aim to stabilize by the application of pulsed stochastic control at each $k$ th step, starting with the step $k-1$. In this article we are not concerned with the case where $L<$ 1 , since it would immediately follow that $\lim _{n \rightarrow \infty} z_{n}=0$, for $\left|z_{0}\right| \leq u_{0}$, and a control is unnecessary.

First, we characterize the control, which may depend on the function $g$, on a deterministic control parameter $\alpha \in[0,1)$, and on a coefficient $l>0$ describing the amplitude of a one-dimensional stochastic perturbation, satisfying Assumption [.2. Note that if $\alpha=0$, any achievable control is due only to this perturbation.

Suppose we apply a general stochastic control to the right-hand side of (5) at the $n=s k-1$ step, for each $s \in$ $\mathbb{N}$, and represent the resulting stochastically controlled map by the function $G: \mathbb{R} \times[0,1] \times\left[0, l_{0}\right] \times[-1,1] \rightarrow \mathbb{R}$ for some $l_{0}>0$. Then the stochastically controlled difference equation becomes (again with $z_{0}>0$ )

$$
z_{n+1}=\left\{\begin{array}{l}
G\left(z_{n}, \alpha, l, \xi_{n+1}\right), \quad n=s k-1, \quad s \in \mathbb{N} \\
g\left(z_{n}\right), \quad \text { otherwise }
\end{array}\right.
$$

Next, we place constraints on the form of the stochastically controlled map $G$ under which we will prove our main result in this section.

Assumption II.2 Define the region $\mathcal{B}:=\{\alpha \in[0,1), l \in$ $\left.\left[0, l_{0}\right],|v| \leq 1\right\}$, and suppose that (6) in the statement of Assumption II.1 holds. There exists a continuous function $\mathcal{L}:[0,1] \times\left[0, l_{0}\right] \times[-1,1] \times[0, \infty) \rightarrow(0, \infty)$ such that

(i) for $(\alpha, l, v) \in \mathcal{B}$,

$$
\begin{aligned}
& |G(z, \alpha, l, v)| \leq \mathcal{L}(\alpha, l, v, u)|z|, \\
& |z| \leq u \leq u_{0}, \quad(\alpha, l, v) \in \mathcal{B}
\end{aligned}
$$

(ii) for some $M>0$

$$
\sup \left\{\mathcal{L}(\alpha, l, v, u):(\alpha, l, v) \in \mathcal{B},|u| \leq u_{0}\right\}=M ;
$$

(iii) for $\xi$ satisfying Assumption II.2, for $L$ as given in (6), for $k$ as given in (77), and for some $\alpha \in[0,1)$, $l \in\left[0, l_{0}\right]$,

$$
\lambda:=-\mathbb{E} \ln \mathcal{L}(\alpha, l, \xi, 0)>(k-1) \ln L .
$$

In our applications, $G$ is the form which right hand side of the equation takes after the shift of the equilibrium to zero, or after some other transformations, and after application of control. The function $\mathcal{L}$ is a local Lipschitz constant of $G$ at zero. Since the control is random, we have both $G$ and $\mathcal{L}$ random. 
Remark II.3 Due to the continuity of $\mathcal{L}$ in $u$, condition (10) implies that for some $u_{1} \in\left(0, u_{0}\right], 0 \leq u \leq u_{1}$,

$$
\lambda(u):=-\mathbb{E} \ln \mathcal{L}(\alpha, l, \xi, u)>(k-1) \ln L .
$$

Inequality (10) is the main assumption of the paper, and it immediately implies (11). The fact that (10), (11) guarantee stability of the zero equilibrium is a consequence of Kolmogorov's Law of Large Numbers. This applies to models that without stochasticity will be unstable, and can be connected to the illustration with Kapica's pendulum ${ }^{22}$. Mathematically, this can be roughly described as possible decrease of Lyapunov exponents by introducing a random component with a zero mean. This approach goes back to H. Kesten in 1960-1970 17,23 , see ${ }^{9}$ for more details. Note that conditions (10) and (11) are quite close to necessary ${ }^{1}$. All the results of the present paper are proved under this condition, control parameters for all the examples are chosen to satisfy this assumption.

Now we present the main result of this section.

Theorem II.4 Let Assumptions I.2, 11.1, 11.2 hold, and let $\gamma \in(0,1)$. Then there exist $\delta_{0}>0$ and $\Omega_{\gamma} \subseteq \Omega$, $\mathbb{P}\left(\Omega_{\gamma}\right)>1-\gamma$, such that for each solution $z$ to Eq. (7) with initial value $\left|z_{0}\right| \leq \delta_{0}$ we have

$$
\lim _{n \rightarrow \infty} z_{n}(\omega)=0, \omega \in \Omega_{\gamma},
$$

where $z_{n}(\omega)$ is a sample path of the solution $z_{n}$. If we additionally suppose that $M L^{k-1}<1$ and $\left|z_{0}\right| \leq \frac{u_{0}}{L^{k-1}}$, then

$$
\lim _{n \rightarrow \infty} z_{n}(\omega)=0, \quad \omega \in \Omega .
$$

Remark II.5 The case $M L^{k-1}<1$ can hold when $G$ is nonrandom, or when $\mathcal{L}$ is nonrandom. In particular it is the case for $P B C$ with a control that is either deterministic or subject to low-intensity stochastic perturbation, see ${ }^{7}$. We discuss this in more detail in Sections $[I A$, IIB: see also Remarks II.19 and II.19. of

In this section we apply Theorem II.4 to stabilize cycles

$$
x_{n+1}=f\left(x_{n}\right), \quad n \in \mathbb{N}_{0}, \quad x_{0}>0 .
$$

First, we specify the structure of the map $f$. Then, we impose upon $f$ stochastic versions of TOC and PBC. The resulting stochastically controlled maps can be converted to form (7). For each model, we then derive assumptions on the control parameter $\alpha$ and the noise intensity $l$, which ensure condition (10) in the statement of Assumption $\amalg .2$ allowing us to apply Theorem II.4

Suppose that $f$ is a real-valued and non-negative function possessing a cycle of period $d \in \mathbb{N}$, and it satisfies a Lipschitz-type condition locally around each point in the cycle:

Assumption II.6 For some $u_{0}>0$ and $d \in \mathbb{N}$, the continuous function $f: \mathbb{R} \rightarrow[0, \infty)$ is such that (a) $f\left(K_{i}\right)=K_{i+1}$ for $i=1, \ldots$, d, where $K_{d+1}:=K_{1}$;

(b) there exist $L_{i}>0, i=1, \ldots, d$, such that

$\left|f(x)-K_{i+1}\right| \leq L_{i}\left|x-K_{i}\right|, x \in\left[K_{i}-u_{0}, K_{i}+u_{0}\right]$.

Remark II.7 If $f$ satisfies Assumption [II.6, it is not necessarily differentiable at $K_{i}$; consider for example $f(x)=|x|$. However, if $f^{\prime}\left(K_{i}\right)$ exists, then for each $u \in\left(0, u_{0}\right)$ and $x \in\left[K_{i}-u, K_{i}+u\right]$, (12) is satisfied with $L_{i}=\left|f^{\prime}\left(K_{i}\right)\right|+\varepsilon(u)$, where $\lim _{u \rightarrow 0} \varepsilon(u)=0$.

Notationally, set $f^{2}(x)=f(f(x)), f^{j}(x)=$ $f\left(f^{j-1}(x)\right), j \in \mathbb{N}$ and note that under Assumption 【I.6. each point of the set $\left\{K_{1}, K_{2}, \ldots, K_{d}\right\}$ is an equilibrium for $f^{d}$. It follows that $f^{d}$ satisfies a generalized Lipschitztype condition locally around each $K_{i}$ :

Lemma II.8 Let Assumption II.6 hold and

$$
L(d):=\prod_{i=1}^{d} \max \left\{1, L_{i}\right\}
$$

Then, for $i=1, \ldots d$,

$$
\left|f^{d}(x)-K_{i}\right| \leq L(d)\left|x-K_{i}\right| \quad \text { for } \quad\left|x-K_{i}\right| \leq \frac{u_{0}}{L(d)} .
$$

Next we assume a Lipschitz-type relationship between the position of $x$ in the vicinity of a point in the $d$-cycle $K_{i}$, and the relative position of $f(x)$ to the next point in the $d$-cycle $K_{i+1}$.

Assumption II.9 For some $u_{0}>0$ and $d \in \mathbb{N}$ the function $f: \mathbb{R} \rightarrow[0, \infty)$ satisfies Part (a) of Assumption $[I .6$. There exist constants $\mathcal{A}_{i} \in \mathbb{R}$ and functions $\phi_{i}: \mathbb{R} \rightarrow \mathbb{R}$, $\psi_{i}: \mathbb{R} \rightarrow[0, \infty), i=1, \ldots, d$, such that

(i) $\psi_{i}(u) \rightarrow 0$ as $u \rightarrow 0$;

(ii) for each $u \in\left(0, u_{0}\right)$ and $x \in\left[K_{i}-u, K_{i}+u\right], i=$ $1,2, \ldots, d$,

$$
\begin{aligned}
f^{i}(x) & =K_{i+1}+\mathcal{A}_{i}\left(x-K_{i}\right)+\phi_{i}(x) ; \\
\left|\phi_{i}(x)\right| & \leq \psi_{i}\left(\left|x-K_{i}\right|\right)\left|x-K_{i}\right| .
\end{aligned}
$$

Since $K_{d+1}=K_{1}$ it follows that $x$ and $f^{d}(x)$ have a similar relationship in the vicinity of $K_{1}$ :

Lemma II.10 Suppose that Assumption II.9 holds, and define for $u \in(0,1)$,

$$
\mathcal{A}(d):=\prod_{i=1}^{d} \mathcal{A}_{i}, u(d):=\frac{u}{\prod_{i=1}^{d} \max \left\{\left|\mathcal{A}_{i}\right|+\psi_{i}(u), 1\right\}} .
$$

Then, there exist functions $\bar{\phi}: \mathbb{R} \rightarrow \mathbb{R}$ and $\bar{\psi}: \mathbb{R} \rightarrow$ $[0, \infty)$ such that for $x \in\left[K_{1}-u(d), K_{1}+u(d)\right]$,

$$
\begin{aligned}
f^{d}(x) & =K_{1}+\mathcal{A}(d)\left(x-K_{1}\right)+\bar{\phi}(x), \\
|\bar{\phi}(x)| & \leq \bar{\psi}\left(\left|x-K_{1}\right|\right)\left|x-K_{1}\right|,
\end{aligned}
$$

where $\bar{\psi}(u) \rightarrow 0$ as $u \rightarrow 0$. 


\section{A. Target-Oriented Control}

Deterministic modified TOC control is characterized in general by Eq. (4). Consider a particular case when the target $T$ coincides with the equilibrium $K$ of $f$, and where the control parameter $\alpha$ is stochastically perturbed by an additive noise of intensity $l$. Then (4) becomes

$$
x_{n+1}=\left(1-\alpha-l \xi_{n+1}\right) f\left(x_{n}\right)+\left(\alpha+l \xi_{n+1}\right) K
$$

for $n \in \mathbb{N}_{0}$. In fact, we apply (4) not for all $n \in \mathbb{N}_{0}$ but at each $k$-th step, and we aim at either a point or a cycle stabilization. To the best of our knowledge, a combination of TOC with either pulsed control, stochastic control, or the use of part of a cycle as a target, is novel and we tackle here all three tasks.

In Sections IA 1 and IA 2. we present equations and conditions for the local stabilization of a point equilibrium and $d$-cycle, respectively, using stochastic TOC. In Section II A 3 we investigate global stabilization.

\section{Pulsed stochastic TOC: stabilization of a point equilibrium.}

Suppose $f(K)=K$, for $K>0$. Consider the stochastic TOC model, pulsed at each $k$ th step, with target $K$ :

$$
\begin{aligned}
& x_{n+1}=\left\{\begin{array}{l}
\left(1-\alpha-l \xi_{n+1}\right) f\left(x_{n}\right) \\
+\left(\alpha+l \xi_{n+1}\right) K \\
n=s k-1, \quad s \in \mathbb{N} \\
f\left(x_{n}\right), \text { otherwise }
\end{array}\right. \\
& \left|x_{0}-K\right|<\delta .
\end{aligned}
$$

If we denote

$$
z_{n}:=x_{n}-K, \quad g(z):=f(z+K)-K,
$$

then (19) takes the form of (7) with

$$
G\left(z_{n}, \alpha, l, \xi_{n+1}\right)=\left(1-\alpha-l \xi_{n+1}\right) g\left(z_{n}\right) .
$$

Assuming that

$$
|f(z+K)-K| \leq L|z|, \quad|z| \leq u_{0}
$$

we get $\mathcal{L}(\alpha, l, v, u)=|1-\alpha-l v| L$, with $M=$ $L \max _{|v| \leq 1}|1-\alpha-l v|$, and condition (10) takes the form

$$
\lambda=-\mathbb{E} \ln |1-\alpha-l \xi|>k \ln L .
$$

Here we also assume that $\xi \neq \frac{1-\alpha}{l}$ in the case when $\xi$ has a discrete distribution, see more details in ${ }^{9}$. The following result now follows directly by an application of Theorem II.4

Theorem II.11 Let Assumption I.2 and conditions (22), (23) hold. Then for each $\gamma \in(0,1)$ there exist $\delta_{0}>0$ and $\Omega_{\gamma} \subset \Omega$ where $\mathbb{P}\left(\Omega_{\gamma}\right)>1-\gamma$, such that for each solution $\left(x_{n}\right)_{n \in \mathbb{N}}$ to equation (19) with initial value satisfying $\left|x_{0}-K\right| \leq \delta_{0}$, we have

$$
\lim _{n \rightarrow \infty} x_{n}(\omega)=0, \quad \omega \in \Omega_{\gamma}
$$

Remark II.12 Note that for $\alpha<1-l$ we have $M=$ $L(1-\alpha+l)$, and condition (23) holds if $1-\alpha+l \leq L^{-k}$, which gives the following ranges for the parameter $\alpha$ and for the noise intensity $l$ to ensure stabilization:

$$
\alpha \in\left(1-L^{-k}, 1\right), \quad l \leq \min \left\{1-\alpha, \alpha-1+L^{-k}\right\} .
$$

For a large value of $L$, the parameter $\alpha$ needs to be close to one, and $l$ needs to be small. In this case, stabilization is due to the deterministic control $\alpha$, and only a small stochastic disturbance is allowed.

It is also possible to demonstrate active stabilization by noise, when $l$ is bigger than in (24). For Bernoulli distributed $\xi$ (taking each of the values \pm 1 with the probability of 0.5), we have $-\lambda=\frac{1}{2} \ln \left|(1-\alpha)^{2}-l^{2}\right|$, and (23) holds if

$$
\sqrt{(1-\alpha)^{2}-L^{-2 k}}<l<\sqrt{(1-\alpha)^{2}+L^{-2 k}}
$$

Note that (25) can remain valid even for large $L$ and $\alpha=0$, but then $l<1$ should be close to 1 .

\section{Pulsed stochastic TOC: stabilization of a $d$-cycle.}

Let $k=m d$ for some $m \in \mathbb{N}$, and let $f$ satisfy Assumption II.6. Recall that each $K_{i}, i=1, \ldots, d$, is a fixed point of $f^{d}$, and therefore of $f^{m d}$. For simplicity we focus only on $K_{1}$, but our analysis applies equally to any other point in the cycle.

Consider the equation

$$
x_{n+1}=\left\{\begin{array}{l}
\left(1-\alpha-l \xi_{n+1}\right) f\left(x_{n}\right)+\left(\alpha+l \xi_{n+1}\right) K_{1}, \\
n=s m d-1, \quad s \in \mathbb{N}, \\
f\left(x_{n}\right), \quad \text { otherwise, } \quad\left|x_{0}-K_{1}\right| \leq \delta .
\end{array}\right.
$$

Set $y_{s}:=x_{(s-1) m d}, \bar{\xi}_{s}:=\xi_{(s-1) m d}$, for $s \in \mathbb{N}$, and note that the sequence $\left(\bar{\xi}_{s}\right)_{s \in \mathbb{N}}$ satisfies Assumption I.2. For $n=s m d-1, s \in \mathbb{N}$, we have

$$
\begin{aligned}
x_{n}=x_{s m d-1}, & x_{n+1}=x_{s m d}=y_{s+1}, \quad y_{1}=x_{0}, \\
f\left(x_{n}\right) & =f^{m d}\left(x_{n-m d+1}\right) \\
& =f^{m d}\left(x_{(s-1) m d}\right)=f^{m d}\left(y_{s}\right) .
\end{aligned}
$$

Thus, (26) can be transformed to

$$
\begin{aligned}
& y_{s+1}=\left(1-\alpha-l \bar{\xi}_{s+1}\right) f^{m d}\left(y_{s}\right)+\left(\alpha+l \bar{\xi}_{s+1}\right) K_{1}, \\
& s \in \mathbb{N}, \quad\left|y_{1}-K_{1}\right| \leq \delta
\end{aligned}
$$

which is in the form of (19) with $k=1, K=K_{1}, f^{m d}$ instead of $f, y_{1}$ instead of $x_{0}$ and $s$ starting from 1 .

Equation (28), in turn, can be transformed to (7) if we set

$$
z_{s}:=y_{s}-K_{1}, \quad g(z):=f^{m d}\left(z+K_{1}\right)-K_{1} .
$$

Note that, by Lemma II.8, $g$ satisfies (6) with constant $L(m d):=L^{m}(d)$ where $L(d)$ is defined by (13). Recall 
that $L(d) \geq 1$. For $G$ defined as in (21), $\mathcal{L}(\alpha, l, v, u)=$ $|1-\alpha-l v| L^{m}(d), M:=(1-\alpha+l) L^{m}(d)$, condition (10) takes the form

$$
\lambda:=-\mathbb{E} \ln |1-\alpha-l \xi|>m \ln L(d) .
$$

Therefore, Theorem ஹ.4 implies $\lim _{s \rightarrow \infty} y_{s}=$ $\lim _{s \rightarrow \infty} x_{(s-1) m d}=K_{1}$, with any given probability $1-\gamma$ and small enough $\delta_{0}$.

To extend this result to show that $\lim _{n \rightarrow \infty} x_{n d+\bar{j}}=$ $K_{\bar{j}}$, for each $\bar{j}=0,1, \ldots, d-1$, we require the next lemma:

Lemma II.13 Let $\left(x_{n}\right)_{n \in \mathbb{N}}$ be a solution of Eq. (26). Let Assumptions [I.2, [I.6, and condition (29) hold. Then for each $\gamma \in(0,1)$ there exist $\delta_{0}>0$ and $\Omega_{\gamma} \subset \Omega$, with $\mathbb{P}\left(\Omega_{\gamma}\right)>1-\gamma$, such that if $\left|x_{0}-K_{1}\right| \leq \delta_{0}$, and $s_{0}$ is such that

$$
\left|x_{s m d}-K_{1}\right|<u_{0} L^{-m}(d)|1-\alpha+l|^{-m+1}, \quad s \geq s_{0},
$$

then for $j=q d+\bar{j}, \bar{j}=0,1, \ldots, d-1, q=0,1, \ldots, m-1$, we have

$\left|x_{s m d+j}-K_{\bar{j}+1}\right| \leq|1-\alpha+l|^{m-1} L^{m}(d)\left|x_{s m d}-K_{1}\right|, s \geq s_{0}$.

All the above brings us to the following theorem:

Theorem II.14 Let $\left(x_{n}\right)_{n \in \mathbb{N}}$ be a solution of Eq. (26). Let Assumptions [.2, 11.6, and condition (29) hold. Then for each $\gamma \in(0,1)$ there exist $\delta_{0}>0$ and $\Omega_{\gamma} \subset \Omega$, with $\mathbb{P}\left(\Omega_{\gamma}\right)>1-\gamma$, such that if $\left|x_{0}-K_{1}\right| \leq \delta_{0}$,

$$
\lim _{n \rightarrow \infty} x_{n d+\bar{j}}(\omega)=K_{\bar{j}}, \quad \omega \in \Omega_{\gamma}, \quad \bar{j}=0,1, \ldots, d-1 .
$$

\section{Global stabilization of a $d$-cycle by stochastic TOC}

Observe that if (22) (when $d=1$, (12) otherwise) holds globally on $\mathbb{R}$, condition (23) (when $d=1$, 29) otherwise) also holds. It is then possible to show that stochastic TOC (19) (respectively (26)) globally stabilizes the equilibrium $K$ (or a $d$-cycle).

Theorem II.15 Theorem II.11 (respectively Theorem II.14) holds for any $x_{0}>0$ if, in conditions (22), (23) (respectively, conditions (12), (29)), local Lipschitz constants are replaced with global Lipschitz constants.

The proof modifies that of Theorem II.4 so that solutions are not required to stay in some neighbourhood of the initial value. Note however that the global Lipschitz constant $\bar{L}$ at the point $K$ (or in the case of a $d$-cycle, $\bar{L}_{i}$ at each $K_{i}, i=1,2 \ldots, d$ ) can be quite large, reaching up to $\sup _{s \in \mathbb{R}}\left|f^{\prime}(s)\right|$. Nonetheless, we will see in Example III.1 that, in the case of Bernoulli $\xi$, condition (23) (respectively (29)) holds for large $\bar{L}\left(\right.$ or $\bar{L}^{m}(d)$ ) even with $\alpha=0$ if $l$ satisfies (25), where we replace $L$ by $\bar{L}$ (or in the case of a $d$-cycle, $L$ is replaced by $\bar{L}^{m}(d)$ and $k$ by $m d)$.

\section{B. Predictive Based Control}

The application of stochastic PBC is characterized in general by Eq. (2). Following the order of investigation in Section IA we will apply pulsed stochastic PBC at each $k$ th step to stabilize a point equilibrium in Section IIB 1, and to stabilize a $d$-cycle in Section IIB2.

The results of this section are illustrated for a point equilibrium in Example III.3, where even local stabilization is not possible for any $\alpha \in(0,1)$ in the absence of a stochastic perturbation, and in Example III.5. where global stabilization is considered. The application of pulsed stochastic PBC to stabilize a 2-cycle is illustrated in Example II.4.

\section{Pulsed stochastic PBC: stabilization of a point equilibrium.}

Suppose $f(K)=K$ for $K>0$, and consider the stochastic PBC model, pulsed at each $k$ th step:

$$
x_{n+1}=\left\{\begin{array}{l}
\left(1-\alpha-l \xi_{n+1}\right) f\left(x_{n}\right)+\left(\alpha+l \xi_{n+1}\right) x_{n}, \\
n=s k-1, s \in \mathbb{N}, \\
f\left(x_{n}\right), \text { otherwise, } \quad\left|x_{0}-K\right| \leq \delta,
\end{array}\right.
$$

which, if we again use notation defined by (20), takes the form of (7), with

$$
G(z, \alpha, l, v):=(1-\alpha-l v) g(z)+(\alpha+l v) z .
$$

We may identify constraints on $f$ that ensure condition (10) holds. Suppose first that for $|x-K| \leq u \leq u_{0}$, $f$ admits expansion (15) with $d=1, K_{i} \equiv K, \mathcal{A}_{i} \equiv \mathcal{A}$, $\phi_{i}(u) \equiv \phi(u)$, and $\psi_{i}(u) \equiv \psi(u)$. In particular, this means that $f$ is differentiable at $K$ with derivative $\mathcal{A}$. Then

$$
\begin{aligned}
& G\left(z_{n}, \alpha, l, \xi_{n+1}\right):= {\left[\left(1-\alpha-l \xi_{n+1}\right) \mathcal{A}+\alpha+l \xi_{n+1}\right] z_{n} } \\
&+\left[1-\alpha-l \xi_{n}\right] \phi\left(z_{n}\right) ; \\
& \mathcal{L}(\alpha, l, v, u):=|(1-\alpha) \mathcal{A}+\alpha+(1-\mathcal{A}) l v| \\
&+|1-\alpha+l| \psi(u) ; \\
& L:=\mathcal{A}+\psi(u),
\end{aligned}
$$

and conditions (10) and (by Remark [1.3) (11) hold if

$$
-\mathbb{E} \ln |(1-\alpha) \mathcal{A}+\alpha+(1-\mathcal{A}) l \xi|>(k-1) \ln |\mathcal{A}| .
$$

The following theorem then follows immediately:

Theorem II.16 Let Assumption I.2. Assumption II.9 with $d=1$, and condition (32) hold, and let $\left(x_{n}\right)_{n \in \mathbb{N}}$ be a solution of (30). Then for each $\gamma \in(0,1)$, there exist $\delta_{0}>0$ and $\Omega_{\gamma} \subset \Omega$ where $\mathbb{P}\left(\Omega_{\gamma}\right)>1-\gamma$, such that, if $\left|x_{0}-K\right| \leq \delta_{0}$, we have

$$
\lim _{n \rightarrow \infty} x_{n}(\omega)=0, \quad \omega \in \Omega_{\gamma} .
$$


Remark II.17 Relation (32) fails if $\mathcal{A}>1$ and $l=0$ for any $\alpha \in(0,1)$ and $k \in \mathbb{N}$. However, the presence of noise with $l>0$ can ensure local stability even for $\alpha=0$ and large $\mathcal{A}$. To see this, assume that $\xi$ is Bernoulli distributed. Then

$$
\lambda=-\frac{1}{2} \ln \left|[(1-\alpha) \mathcal{A}+\alpha]^{2}-(1-\mathcal{A})^{2} l^{2}\right|,
$$

and (32) holds if

$$
\begin{aligned}
l_{\text {low }} & :=\frac{[(1-\alpha) \mathcal{A}+\alpha]^{2}-\mathcal{A}^{-2(k-1)}}{(1-\mathcal{A})^{2}}<l^{2} \\
& <\frac{[(1-\alpha) \mathcal{A}+\alpha]^{2}+\mathcal{A}^{-2(k-1)}}{(1-\mathcal{A})^{2}} .
\end{aligned}
$$

If $\alpha=0$ and $k=1$, the lower bound on $l$ is given by $l_{\text {low }}=\frac{\mathcal{A}^{2}-1}{(1-\mathcal{A})^{2}}=1+\frac{2}{\mathcal{A}-1}>1$, for each $\mathcal{A}>1$. For example, if $\mathcal{A}=2, \alpha=0$, and $k=1$ we required $1.73 \approx \sqrt{3}<l<\sqrt{5}$ for (32) to hold. So it is reasonable to combine a nonzero control parameter $\alpha$ with nonzero noise intensity $l$.

Note that for $k=1$ and any $\mathcal{A} \neq 1$ the lower bound on $l$ satisfies

$$
l_{\text {low }}=\frac{(1-\alpha)[(1-\alpha)(\mathcal{A}-1)+2]}{\mathcal{A}-1} \rightarrow 0, \quad \text { as } \alpha \rightarrow 1,
$$

while for $k>1$

$$
\begin{gathered}
l_{\text {low }}=\frac{[(1-\alpha)(\mathcal{A}-1)+1]^{2}-1}{(1-\mathcal{A})^{2}}+\frac{1-\mathcal{A}^{-2(k-1)}}{(1-\mathcal{A})^{2}} \\
\rightarrow \frac{1-\mathcal{A}^{-2(k-1)}}{(1-\mathcal{A})^{2}} \text { as } \alpha \rightarrow 1 .
\end{gathered}
$$

Therefore, when $k=1$ and $\mathcal{A} \neq 1$, for any $\varepsilon>0$ we can choose $\alpha \in(0,1)$ such that the $l_{\text {low }}$ satisfies $l_{\text {low }}<\varepsilon$. In other words, small noise stabilizes the equilibrium if $\alpha$ is close to 1 . When $k>1$ and $\mathcal{A}>2$,

$$
\frac{1-\mathcal{A}^{-2(k-1)}}{(1-\mathcal{A})^{2}}<\frac{1}{(1-\mathcal{A})^{2}}<1,
$$

so there exists $\alpha \in(0,1)$ such that $l_{\text {low }}<1$.

We can relax the assumption that $f$ is differentiable at $K$, instead requiring only that $f(x)-K$ changes sign from positive to negative as $x$ increases through some neighbourhood of $K$. This corresponds to the case $\mathcal{A}<$ -1 if $f$ is differentiable at $K$.

Theorem II.18 Let $\left(x_{n}\right)_{n \in \mathbb{N}}$ be a solution to (30) where (22) holds and suppose that $f(x)>K$ for $x \in\left[K-u_{0}, K\right]$, $f(x)<K$ for $x \in\left[K, K+u_{0}\right]$, and $l \in(0, \min \{\alpha, 1-\alpha\})$. (i) If either

$$
k=1, \quad \alpha>1-L^{-1}, \quad l<L^{-1}-1+\alpha,
$$

or

$$
\begin{gathered}
k>1,1<L^{k}<L+1, \alpha \in\left(1-L^{-k}, L^{-k+1}\right), \\
l \in\left(0, \min \left\{L^{-k}-1+\alpha, \quad L^{-k+1}-\alpha\right\}\right), \\
\text { then for }\left|x_{0}-K\right| \leq \delta \leq u_{0} / L^{k}, \\
\quad \lim _{n \rightarrow \infty} x_{n}(\omega)=K, \quad \text { for all } \omega \in \Omega .
\end{gathered}
$$

(ii) If

$$
\begin{aligned}
\lambda:= & -\mathbb{E} \max \{\ln (|\alpha+l \xi|), \ln (|1-\alpha-l \xi| L)\} \\
& >(k-1) \ln L,
\end{aligned}
$$

then, for any $\gamma \in(0,1)$, there exist $\delta>0$ and $\Omega_{\gamma} \subset$ $\Omega$ with $\mathbb{P}\left(\Omega_{\gamma}\right)>1-\gamma$, such that, for $\left|x_{0}-K\right| \leq \delta$, we have

$$
\lim _{n \rightarrow \infty} x_{n}(\omega)=K, \quad \omega \in \Omega_{\gamma} .
$$

Remark II.19 In Theorem 11.18, part (i) describes a situation where stabilization is due to the action of the underlying deterministic control, and the noise intensity $l$ is kept small to preserve this effect. The case of $k=1$ in part (i) of Theorem 11.18 where (33) holds was covered in $\underline{\underline{Z}}$, and this analysis included establishing the global stability. However, for local stability Theorem 11.18 generalizes the results of $\underline{7}$ to the case where the noise plays an active role in achieving stability: it applies to the situations where, for a chosen $\alpha \in(0,1)$ and $l=0$, the point equilibrium is unstable. The use of pulsed control here to achieve stabilization is also novel.

In part (ii) the noise also plays an active role, and (35) gives a set of stabilizing parameters different from those in part (i). Here we present an example where (35) is fulfilled, deferring a more detailed description and illustrative numerical simulation until Example III.5.

The inequality $\mathbb{E} \ln [1-\alpha-l \xi]<k \ln L$ implies (35) if $(1-\alpha-l v) L \geq \alpha+l v$ holds for each $|v| \leq 1$. The latter is true when $\alpha+l<1-(1+L)^{-1}$. In the case of Bernoulli distributed $\xi$, this gives a lower bound for $l^{2}$ as $l_{\text {low }}=(1-\alpha)^{2}-L^{-2 k}$. It can be shown that the Ricker model with $r=2.41, L=1.5$, and the control with $\alpha=0.3, l=0.24, k=1$, satisfies (35) in part (ii) but not (33) in part (i) of Theorem II.18.

Suppose more specifically that $(1-\alpha-l v) L=\alpha+l v$ for some $v \in(-1,1)$, and $\alpha+l>1-(L+1)^{-1}$. Then in order to satisfy (35) we need $\alpha(1-\alpha)+l(1+l)<L^{-k}$. It can be shown that for the Ricker model with $r=2.2$, the values $L=1.2, \alpha=0.28, l=0.27, k=2$ satisfy (35) in part (ii), but not (34) in part (i) of Theorem II.18, More details may be found in Example III.5.

Remark II.20 There are cases (applicable to both Ricker and logistic models) for which local stability implies global stability. Suppose $k=1$, so that control is 
applied at every step. Then, in the deterministic case, we have

$$
\begin{aligned}
& f_{\alpha}(x):=(1-\alpha) x e^{r(1-x)}+\alpha x, \\
& f_{\alpha}^{\prime}(x)=(1-\alpha)(1-r x) e^{r(1-x)}+\alpha .
\end{aligned}
$$

The controlled map $f_{\alpha}$ is unimodal with a negative Schwarzian derivative, and so equilibria of the controlled deterministic equation are globally stable once they are locally stable. The general form of this result is due to Singer 35 , see also ${ }^{24}$. For deterministic $P B C$ the result is $i n^{25}$, and some extensions of the idea can be found in 16 . The point equilibrium $K=1$ for the Ricker model is locally stable if

$$
f_{\alpha}^{\prime}(1)=(1-\alpha)(1-r)+\alpha>-1
$$

or $\alpha \in\left(\alpha^{*}, 1\right)$, where $\alpha^{*}=(r-2) / r$. According to ${ }^{7}$, stabilization is achieved once $(\alpha-l, \alpha+l) \subseteq\left(\alpha^{*}, 1\right)$.

\section{Pulsed stochastic PBC: stabilization of a $d$-cycle.}

Suppose that $k=m d, m \in \mathbb{N}$, and Assumption \.9 holds. Consider the equation

$$
x_{n+1}=\left\{\begin{array}{l}
\left(1-\alpha-l \xi_{n+1}\right) f\left(x_{n}\right) \\
+\left(\alpha+l \xi_{n+1}\right) x_{n-m d+1}, \quad\left|x_{0}-K_{1}\right| \leq \delta . \\
n=s m d-1, \quad s \in \mathbb{N}, \\
f\left(x_{n}\right), \quad \text { otherwise. }
\end{array}\right.
$$

To this model, we apply transformation (27) using notation as in Section I A 2 to get

$$
\begin{gathered}
y_{s+1}=\left(1-\alpha-l \bar{\xi}_{s+1}\right) f^{m d}\left(y_{s}\right)+\left(\alpha+l \bar{\xi}_{s+1}\right) y_{s}, \\
s \in \mathbb{N}, \quad\left|y_{1}-K_{1}\right| \leq \delta
\end{gathered}
$$

which is covered by the case discussed in Section IIB 1. To see this, substitute $f^{d m}$ for $f$, and $\bar{\xi}_{s}:=\xi_{s d m}$ for $\xi_{n}$ and $k=1$. Note that, by Lemma II.10, $f^{m d}$ admits the expansion (17), substituting $m d$ for $d$ and $\mathcal{A}(m d)=$ $\mathcal{A}^{m}(d)$, where $\mathcal{A}(d)$ is defined as in (16). Therefore condition (10) has the form

$$
-\mathbb{E} \ln \left|(1-\alpha) \mathcal{A}^{m}(d)+\alpha+\left(1-\mathcal{A}^{m}(d)\right) l \xi\right|>0 .
$$

Following the arguments of Section II 2, we obtain the following theorem.

Theorem II.21 Let $\left(x_{n}\right)_{n \in \mathbb{N}}$ be a solution of Eq. (36). Let Assumptions I.2, II.9, and condition (37) hold. Then for each $\gamma \in(0,1)$ there exist $\delta_{0}>0$ and $\Omega_{\gamma} \subset \Omega$, with $\mathbb{P}\left(\Omega_{\gamma}\right)>1-\gamma$, such that if $\left|x_{0}-K_{1}\right| \leq \delta_{0}$,

$$
\lim _{n \rightarrow \infty} x_{n d+\bar{j}}(\omega)=K_{\bar{j}}, \quad \omega \in \Omega_{\gamma}, \quad \bar{j}=0,1, \ldots, d-1 .
$$

\section{EXAMPLES AND COMPUTER SIMULATIONS}

In all simulations presented in this Section, we truncate the controlled map

$$
x_{n+1}=\max \left\{\left(1-\alpha-l \xi_{n+1}\right) f\left(x_{n}\right)+\left(\alpha+l \xi_{n+1}\right) K, 0\right\}
$$

in order to avoid negative values $x_{n}$.

We illustrate the results of Sections $\llbracket \mathrm{A}$ and $\Pi \mathrm{B}$ using difference equations associated with the Ricker function

$$
f_{1}(x)=x e^{r(1-x)}, \quad x \geq 0
$$

the logistic map

$$
f_{2}(x)=r x(1-x), \quad x \geq 0,
$$

and the Maynard Smith model ${ }^{36}$ with

$$
f_{3}(x)=\frac{3 x}{2+(x-3)^{2}}, \quad x \geq 0 .
$$

Here we simulate continuous uniformly distributed on $[0,1]$ and Bernoulli random variables $\xi_{n}$ to illustrate some cases from Sections $\amalg \mathrm{A}$ and IIB. Each plot incorporating stochastic perturbations $(l>0)$ was generated with 3 runs, with a single run used to generate deterministic plots.

We start with the TOC method. Examples III.1 and III.2 illustrate Theorem II.21, $d=2$, applied to Ricker and logistic functions.

Example III.1 First, we consider stochastic TOC (19) applied at alternate steps $(k=2)$ to a chaotic Ricker map $f_{1}$ satisfying (38) with $r \approx 3.2716$ and a continuous uniformly distributed on $[-1,1]$ noise. Without noise, $\alpha=0.7$ guarantees pulsed cycle stabilization, while uniformly distributed noise with $l=0.4$ leads to stabilization of $K=1$, see Fig. 1 .

Next, apply a Bernoulli noise with a smaller $\alpha=0.3$ to stabilize 2-cycle $K_{1}=0.1, K_{2}=1.9$ using $K_{1}$ as the target. Fig. 2, right, presents stabilization for noise intensity $l=0.7$. For $l=0.7$, Fig. 2, left illustrates that there is no convergence to this 2-cycle, and similar results are obtained for $l>0.75$, the range of values of $l$ that allow stabilization is quite narrow.

Example III.2 Consider stochastic TOC (19) applied at alternate steps $(k=2)$ to a logistic map $f_{2}$ satisfying (39) with $r=3.5$. We can globally stabilize an unstable 2-cycle. Fig. 3 shows convergence with $\alpha=0, r=3.5$, $l=1.2$. For significatly smaller $l$, there is no convergence, and the effective stabilizing range for $l$ is narrow. Increasing to $\alpha=0.2$ in Fig. 4 leads to a higher convergence speed, see Fig. 4 for fast stabilization of a 2-cycle for $l=0.75$ and $l=0.8$.

Now we proceed to PBC method. Examples III.3 and III.4 illustrate Theorem I.21, $m=1$, with $d=1$ and $d=2$, respectively. 

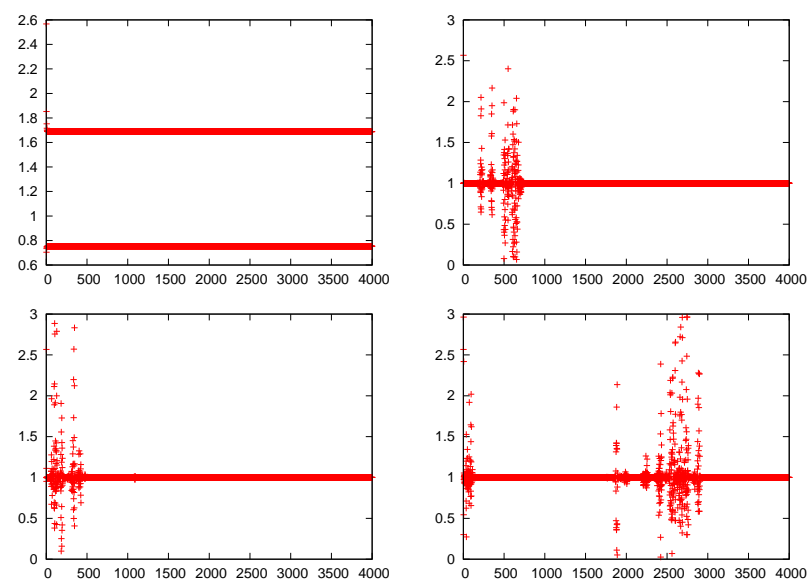

FIG. 1. Model (19) with $f=f_{1}$ from (38) with $r \approx 3.2716$, $\alpha=0.7, m=2, d=1, x_{0}=0.5$ and (top, left) no noise, (top, right) $l=0.32$, (bottom, left) $l=0.4$ and (bottom, right) $l=0.42$.
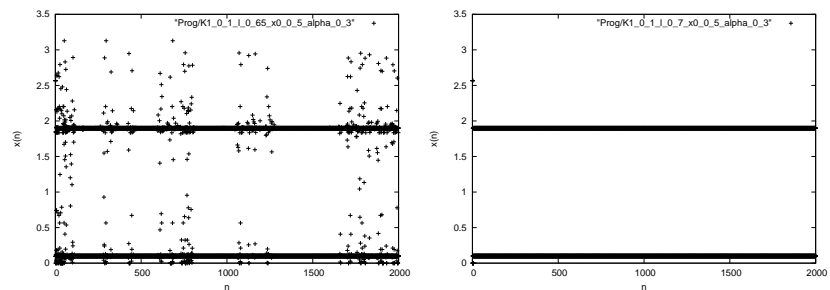

FIG. 2. Model (26) with $f=f_{1}$ from (38) with $r \approx 3.2716$, $\alpha=0.3, m=1, d=2, x_{0}=0.5$, and $l=0.65,0.7$.

Example III.3 Consider stochastic PBC (30) applied at every step to a Maynard-Smith model $f_{3}$ satisfying (40) and note that this model is chaotic for $\alpha=0, l=0$. There are two positive equilibria at $x=2$ and $x=4$, and $f_{3}^{\prime}(2)=\frac{7}{3}>1$. Note also that even local stabilization is not possible for any $\alpha \in(0,1)$ in the absence of noise $(l=0)$. Fig. $[5$ illustrates local stabilization of the equilibrium $x \equiv 2$ with $\alpha=0.8$ and $l=0.4,0.5,0.7$. The solution $x \equiv 4$ is stable for $l=0$, and there is no stabilization of $x \equiv 2$ for $l=0.4$, we observe wandering between the two equilibria for $l=0.5$ and stabilization of $x \equiv 2$ for $l=0.7$.

Example III.4 Let us illustrate Theorem III.21 for $d=$ 2, $m=1$. Applying pulsed stochastic PBC to stabilize a 2-cycle, we consider (36), "delayed" stabilization, applied to a Ricker map $f_{1}$ satisfying (38) with $r=3.2$, $\alpha=0.4$. Fig. [6 shows how an appropriately chosen noise intensity $l$ leads to global stabilization of the 2 -cycle $\left\{K_{1} \approx 0.11, K_{2} \approx 1.89\right\}$, and l changing from zero (no noise) to $l=0.45$.

The next example illustrates Theorem $\llbracket .18$.

Example III.5 Consider stochastic PBC (30) applied at each step to a Ricker map $f_{1}$ satisfying (38) with
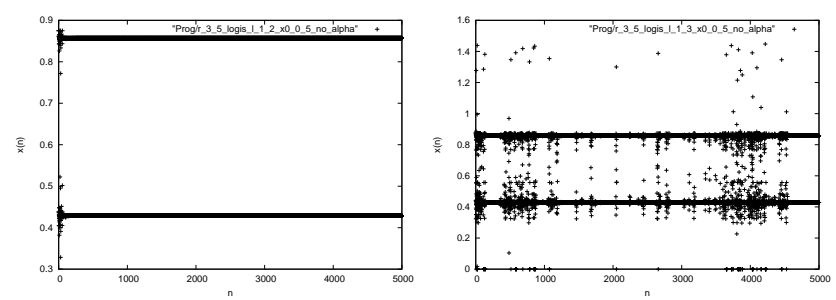

FIG. 3. Model (19) with $f=f_{2}$ from (39), $r=3.5, \alpha=0$, $m=1, d=2, x_{0}=0.5$, and (from left to right) $l=1.2,1.3$.
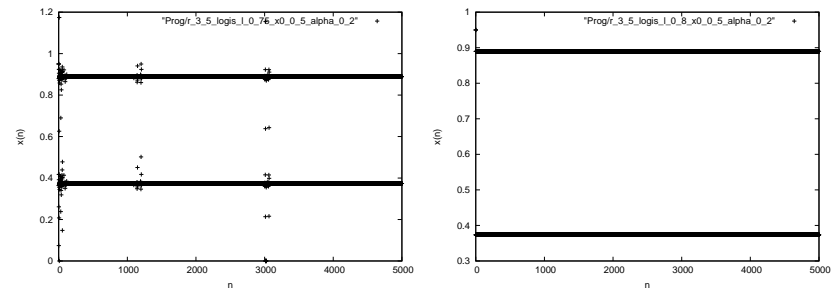

FIG. 4. Model (19) with $f=f_{2}$ from (39), $\alpha=0.2, m=1$, $d=2, x_{0}=0.5$ and (from left to right) $l=0.75,0.8$.

$r=2.41$. The global Lipschitz constant is $L=1.5$. Note that according to Remark II.20, for $r=2.41$ we get

$$
\begin{gathered}
f_{\alpha}^{\prime}(1)=-1.41(1-\alpha)+\alpha=2.41 \alpha-1.41>-1 \\
\Leftrightarrow \alpha>\frac{0.41}{2.41} \approx 0.17012448 .
\end{gathered}
$$

Thus the stabilization bound is $\alpha^{*}=0.1701245$, and $b y^{\underline{7}}$, stabilization is achieved once $\alpha-l>\alpha^{*}, \alpha+l<1$. For $\alpha=0.3, l=0.24$, the first inequality is not satisfied $\alpha-l=0.06<\alpha^{*}$, so our previous result in $n^{7}$ does not allow us to establish stability of the controlled model. It is possible to illustrate global stabilization of the equilibrium $K=1$ with $k=1, \alpha=0.3$ and $l=0.24$.

We can also make $K=1$ stable with a stochastic pulsed control. Global stabilization by pulsed stochastic PBC (30) applied at alternate steps $(k=2)$ to a Ricker map $f_{1}$ satisfying (38) with $r=2.2$ is demonstrated in Fig. 7. Only local stability conditions from Theorem II.16 hold, but global stability is observed. This indicates a possible direction for future research.

\section{CONCLUSIONS}

\section{A. Summary of results}

We have presented a general framework for stabilization of iterative systems by the application of control. The method demonstrates how the effective range of control parameters can be extended by stochastic perturbation.

Our results may be summarized as follows: 

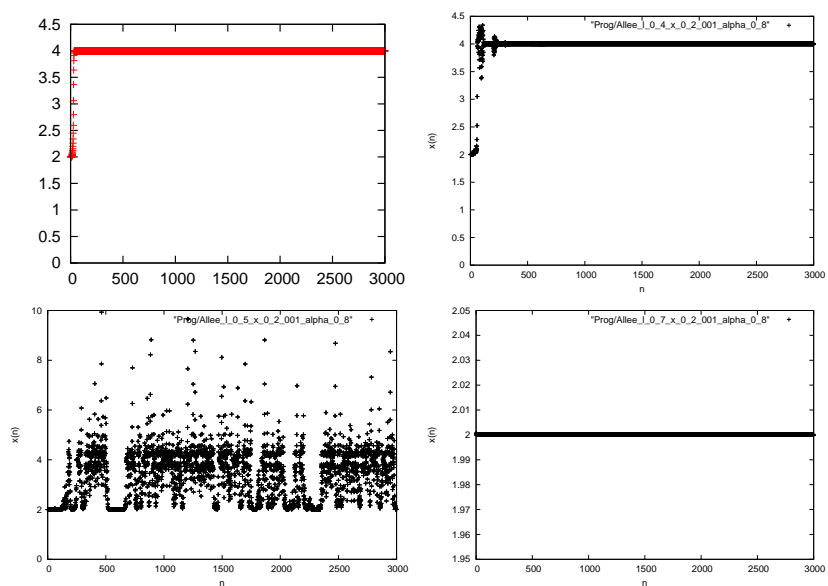

FIG. 5. Model (30) with $f=f_{3}$ from (40), $k=1, \alpha=0.2$, $x_{0}=2.001$ and (top) $l=04, l=0.4$ and (bottom) $l=0.5$, $l=0.7$.
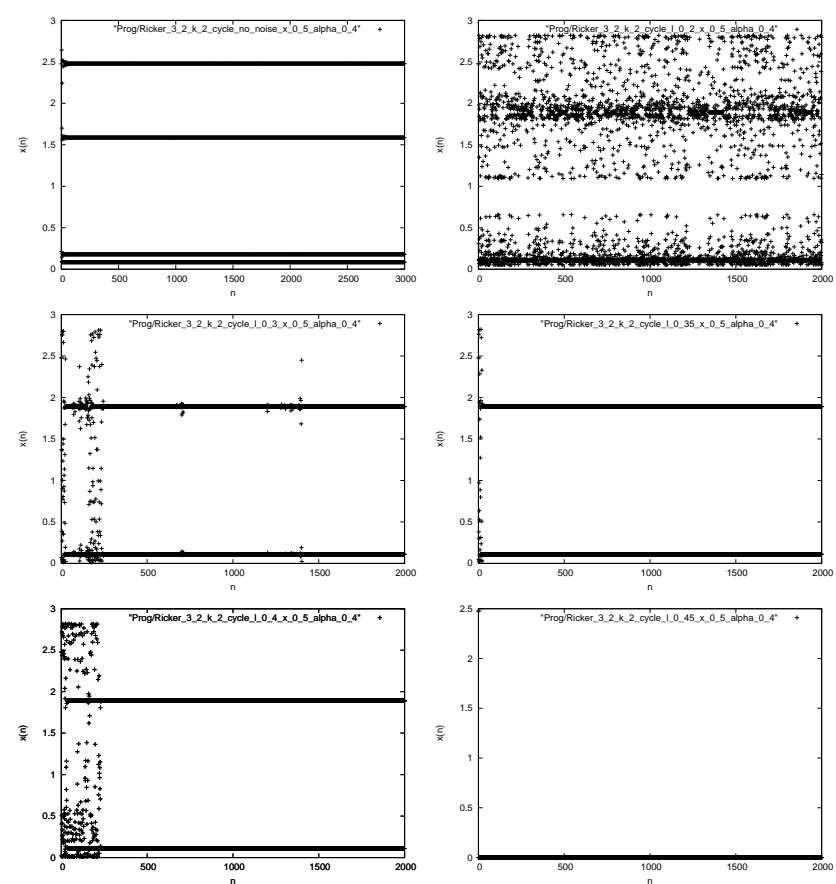

FIG. 6. Model (36) with $f=f_{1}$ from (38) with $r=3.2$, $k=2, \alpha=0.4, x_{0}=0.5$ and (from left to right, top to bottom) $l=0,0.2,0.3,0.35,0.4,0.45$.

- We present a broad general characterization of control that allows us to consider mechanisms incorporating both deterministic and stochastic components. This characterization includes stochastic forms of Prediction-Based Control and TargetOriented Control; the latter is considered here for the first time.

- Both regular (applied at each step) and pulsed (applied every $k$ th step) types of control were investigated. Pulsed TOC applied to one-dimensional
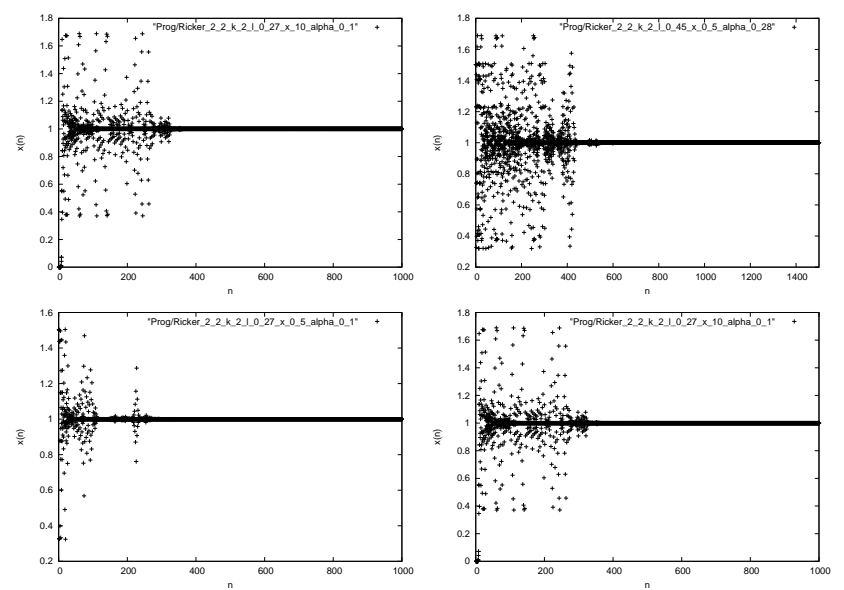

FIG. 7. Model (30) with $f=f_{1}$ from (38), $k=2, r=2.2$. and (top left) $\alpha=0.28, l=0.27, x_{0}=0.5$, (right) $l=0.45$, $x_{0}=0.5$, (bottom) $\alpha=0.1, l=0.27$, (left) $x_{0}=0.5$, (right) $x_{0}=10$.

models has not been studied before. Further, while pulsed stabilization of an equilibrium or a cycle by deterministic control has attracted some attention (see for example ${ }^{8,26}$ ), the use of stochastic PBC and TOC for $k$-cycle stabilization is novel.

- The analysis extends that of ${ }^{7}$, which also showed how the effective range of stabilizing control parameters may be extended by the introduction of noise. However, in this article we additionally explore pulsed stochastic control and the stabilization of unstable $k$-cycles.

\section{B. Future directions for research}

Future research will follow one (or more) of the four directions:

1. Study of sharp and/or global stabilization conditions. Note that some of our results are essentially local, and we observe this in simulation. Nevertheless, we believe it is still possible to get global stabilization results for the PBC method if we introduce some additional restrictions on $f$ and choose the noise intensity appropriately. Example III.5 in fact illustrates the global stabilization for a wide range of parameters, however theoretical justification is yet to be obtained. Also, the conditions for stabilization presented in this article are sufficient but not necessary. It is desirable to obtain necessary and sufficient conditions, as are available in the deterministic setting: see $\frac{15,24,25}{}$.

2. Explore the dependency of parameter bounds on the noise distribution type. Most of our examples assume Bernoulli-distributed noise 
perturbation. Creating a library of sufficient estimates for control parameters and noise amplitudes under various types of noise distribution would be interesting and useful. For stabilization with noise only, such results can be found in ${ }^{10}$.

3. Generalization of scalar results to systems or higher-order difference equations. $\operatorname{In}^{5}$ and $^{6}$, stabilization of high-order and vector difference equations was considered. This allowed to analyse stage-structured and delayed population dynamics models. Even without formal control, the introduction of noise can improve population dynamics, for example, reduce oscillation amplitudes. The next stage of research is to incorporate the ideas and methods of the present paper in the controlled systems of difference equations. For example, if a system describes a network, together with stabilization of periodic orbits ${ }^{26}$, the problem of synchronization is of importance, especially if there are delays and/or stochastic component in communications 28,29 .

4. Qualitative analysis of positive effect of noise: ecological perspective. Finally, it would be interesting, similarly to the present paper, to qualitatively evaluate possible positive effect of noise on stability and population survival, see ${ }^{32}$ for analysis of population interactions and the influence of stochasticity on survival, extinction, and coexistence.

\section{ACKNOWLEDGMENTS}

The authors are grateful to Prof. Gregory Berkolaiko for the fruitful discussion of application of the Law of Large Numbers to PBC method and to two anonymous reviewers whose thoughtful and valuable comments significantly contributed to the present form of the paper. The first author was partially supported by the NSERC research grant RGPIN-2020-03934.

\section{DATA AVAILABILITY STATEMENT}

The data that support the findings of this study are available from the corresponding author upon reasonable request.

\footnotetext{
${ }^{1}$ J. A. D. Appleby, G. Berkolaiko and A.Rodkina, Non-exponential stability and decay rates in nonlinear stochastic difference equations with unbounded noise, Stochastics 81 (2009), 99-127.

${ }^{2}$ I. Bashkirtseva, Mean-square analysis of stochastic cycles in nonlinear discrete-time systems with parametric noise, J. Difference Equ. Appl. 20 (2014), 1178-1189.

${ }^{3}$ I. Bashkirtseva, E. Ekaterinchuk and L. Ryashko, Attractors of randomly forced logistic model with delay: stochastic sensitivity and noise-induced transitions, J. Difference Equ. Appl. 22 (2016), 376--390.
}

${ }^{4}$ E. Braverman and B. Chan, Stabilization of prescribed values and periodic orbits with regular and pulse target oriented control. Chaos, 24(1):013119, 2014.

${ }^{5}$ E. Braverman and D. Franco, Stabilization with target oriented control for higher order difference equations. Physics Letters A 379(16) (2015), 1102-1109.

${ }^{6}$ E. Braverman and D. Franco, Stabilization of structured populations via vector target-oriented control, Bull. Math. Biol. 79 (2017), 1759--1777.

${ }^{7}$ E. Braverman, C. Kelly and A. Rodkina, Stabilisation of difference equations with noisy prediction-based control, Physica D 326 (2016), 21-31.

${ }^{8}$ E. Braverman and E. Liz, On stabilization of equilibria using predictive control with and without pulses. Comput. Math. Appl. 64 (2012), 2192-2201.

${ }^{9}$ E. Braverman and A. Rodkina, Stochastic control stabilizing unstable or chaotic maps, J. Difference Equ. Appl. 25 (2019), 151-178.

${ }^{10}$ E. Braverman and A. Rodkina, Global stabilization and destabilization by the state dependent noise with particular distributions, Physica D 403 (2020), 132302, https://doi.org/10.1016/j.physd.2019.132302.

${ }^{11}$ J. Calatayud, J.-C. Cortés, M. Jornet and L. Villafuerte, Random non-autonomous second order linear differential equations: mean square analytic solutions and their statistical properties, Adv. Difference Equ. 2018, Paper No. 392, 29 pp.

${ }^{12}$ J.-C. Cortés, A. Navarro-Quiles, J.-V. Romero and M.-D. Roselló, Full solution of random autonomous first-order linear systems of difference equations. Application to construct random phase portrait for planar systems, Appl. Math. Lett. 68 (2017), 150-156.

${ }^{13}$ J. Dattani, J. C. Blake, and F. M. Hilker, Target-oriented chaos control, Phys. Lett. A 375(2011), 3986-3992.

${ }^{14}$ N. H. Du and N. T. Dieu, Stochastic dynamic equations on time scales, Acta Math. Vietnam. 38 (2013), 317--338.

${ }^{15}$ D. Franco and E. Liz, A two-parameter method for chaos control and targeting in one-dimensional maps, Internat. J. Bifur. Chaos Appl. Sci. Engrg. Int. 23 (2013), 1350003, 11pp.

${ }^{16}$ D. Franco, J. Perán and J. Segura, Stability for one-dimensional discrete dynamical systems revisited, Discrete Contin. Dyn. Syst. Ser. B 25 (2020), 635-650.

${ }^{17} \mathrm{H}$. Furstenberg and H. Kesten, Products of random matrices, Ann. Math. Statist. 31 (1960), 457-469.

${ }^{18} \mathrm{G}$. Guzik, Asymptotic stability of discrete cocycles, J. Difference Equ. Appl. 21 (2015), 1044--1057.

${ }^{19}$ R. Z. Has'minski, Stability of Systems of Differential Equations under Random Perturbations of Their Parameters, Nauka, Moscow, 1969, 367 pp.

${ }^{20} \mathrm{P}$. Hitczenko and G. Medvedev, Stability of equilibria of randomly perturbed maps, Discrete Contin. Dyn. Syst. Ser. B 22 (2017), 269-281.

${ }^{21}$ R. Kadiev and P. Simonov, Initial data stability and admissibility of spaces for Itô linear difference equations, Math. Bohem. 142 (2017), 185-196.

22 P. P. Kapica, Dynamical stability of pendulum with vibrating suspension, Journal of Experimental and Theoretical Physics 21 (1951), 588-597 (in Russian).

${ }^{23}$ H. Kesten, Random difference equations and renewal theory for the product of random matrices, Acta Math. 131 (1973), 207248.

${ }^{24}$ E. Liz, Local stability implies global stability in some onedimensional discrete single-species models, Discrete Contin. Dyn. Syst. Ser. B 7 (2007), 191-199.

${ }^{25}$ E. Liz and D. Franco, Global stabilization of fixed points using predictive control, Chaos 20 (2010), 023124, 9 pages.

${ }^{26} \mathrm{E}$. Liz and C. Pötzsche, PBC-based pulse stabilization of periodic orbits, Physica D 272 (2014), pp. 26-38.

${ }^{27} \mathrm{G}$. Manjunath and H. Jaeger, The dynamics of random difference equations is remodeled by closed relations, SIAM J. Math. Anal. 46 (2014), 459-483. 
${ }^{28}$ M. Nag and S. Poria, Synchronization in a network of delay coupled maps with stochastically switching topologies, Chaos Solitons Fractals 91 (2016), 9-16.

${ }^{29} \mathrm{M}$. Porfiri and I. Belykh, Memory matters in synchronization of stochastically coupled maps, SIAM J. Appl. Dyn. Syst. 16 (2017), 1372-1396.

${ }^{30}$ L. I. Rodina, On repelling cycles and chaotic solutions of difference equations with random parameters (Russian), Tr. Inst. Mat. Mekh. 22 (2016), 227--235.

${ }^{31} \mathrm{~S}$. J. Schreiber, Chaos and population disappearances in simple ecological models, J. Math. Biol. 42 (2001), 239-260.

${ }^{32}$ S. J. Schreiber, Coexistence in the face of uncertainty, Recent progress and modern challenges in applied mathematics, modeling and computational science, 349-384, Fields Inst. Commun., 79, Springer, New York, 2017.

${ }^{33}$ L. Shaikhet, Lyapunov Functionals and Stability of Stochastic Difference Equations, Springer-Verlag, London, 2011.

${ }^{34}$ A. N. Shiryaev. Probability. 2nd edition, Springer, Berlin, 1996.

${ }^{35}$ D. Singer, Stable orbits and bifurcation of maps of the interval, SIAM J. Appl. Math. 35 (1978), 260-267.

${ }^{36}$ H.R. Thieme, Mathematics in Population Biology, Princeton University Press, Princeton, 2003.

${ }^{37} \mathrm{~T}$. Ushio and S. Yamamoto, Prediction-based control of chaos, Phys. Lett. A 264 (1999), 30-35.

\section{Proof of Theorem}

Consider two cases in turn: (a) $M L^{k-1}<1$ and (b) $M L^{k-1} \geq 1$.

Case (a): If $M L^{k-1}<1$ then (11) holds for $u_{1}=u_{0}$. Since Eq. (6) in Assumption [1.1 holds with $L \geq 1$, we must have $M<1$. Fix $u \in\left(0, u_{0}\right]$ and set $\delta_{0} \leq u / L^{k-1}$. Then, for $\left|z_{0}\right| \leq \delta_{0}$,

$$
\begin{aligned}
\left|z_{1}\right| & \leq L\left|z_{0}\right| \leq \frac{u}{L^{k-2}} \leq u, \\
\left|z_{2}\right| & \leq L\left|z_{1}\right| \leq L^{2}\left|z_{0}\right|<\frac{u}{L^{k-3}} \leq u, \\
& \vdots \\
\left|z_{k-1}\right| & \leq L\left|z_{k-2}\right| \leq L^{k-1}\left|z_{0}\right| \leq u, \\
\left|z_{k}\right| & \leq M\left|z_{k-1}\right| \leq M L^{k-1}\left|z_{0}\right| \leq M u<u, \\
\left|z_{2 k}\right| & \leq\left(M L^{k-1}\right)^{2} u \leq u .
\end{aligned}
$$

Reasoning recursively, we conclude that, for $i \in \mathbb{N}$,

$$
\begin{gathered}
\left|z_{i k}\right| \leq\left(M L^{k-1}\right)^{i} u \leq u, \\
\left|z_{i k+j}\right| \leq\left(M L^{k-1}\right)^{i} u \leq u, j=1, \ldots, k-1,
\end{gathered}
$$

where $M L^{k-1}<1$, so, $\lim _{i \rightarrow \infty}\left|z_{i k+j}(\omega)\right|=0$ for all $\omega \in \Omega$, which concludes the proof of Part (a).

Case (b): Let $\alpha$ and $l$ be chosen as in (10), and $u_{1}$ be defined as in (11). Fix $u \leq u_{1}$ and $\gamma \in(0,1)$.

Applying Lemma I.1 and condition (11) we conclude that, for $\varepsilon:=1 / 2[\lambda-(k-1) \ln L]>0$, there exists a random $\mathcal{N}=\mathcal{N}(\gamma, \lambda, u)$ such that

$$
\left|\frac{1}{n} \sum_{i=0}^{n} \ln \mathcal{L}\left(\alpha, \xi_{i k}, u\right)+\lambda\right|<\varepsilon, \quad n \geq \mathcal{N} .
$$

Then there exist a nonrandom $N=N(\gamma, \lambda, u)$ and $\Omega_{\gamma} \subset$ $\Omega$ with $\mathbb{P}\left(\Omega_{\gamma}\right)>1-\gamma$, such that

$$
\begin{aligned}
& \prod_{i=0}^{n} \mathcal{L}\left(\alpha, l, \xi_{i k}(\omega), u\right) \\
< & \exp \left\{-\frac{n}{2}(\lambda+(k-1) \ln L)\right\}, n \geq N, \omega \in \Omega_{\gamma} .
\end{aligned}
$$

Suppose that, when $N$ is chosen so that A.1 holds, $\delta_{0}$ satisfies

$$
\delta_{0} \leq \frac{u}{\left(\bar{M} L^{k-1}\right)^{N}}, \quad \text { where } \quad \bar{M}:=\max \{1, M\} .
$$

Since $\bar{M} \geq 1$, we have, for all $i=1, \ldots, k-1$,

$$
\begin{aligned}
& \bar{M}^{N} L^{N(k-1)-i}=\bar{M}^{N}\left(L^{k-1}\right)^{N-\frac{i}{k-1}} \\
\geq & \bar{M}^{N-i / k-1}\left(L^{k-1}\right)^{N-\frac{i}{k-1}} \geq 1 .
\end{aligned}
$$

By (6), (77) and (A.2) we have, for $\left|z_{0}\right| \leq \delta_{0} \leq u$,

$$
\left|z_{1}\right|=\left|g\left(z_{0}\right)\right| \leq \frac{L u}{\left(\bar{M} L^{k-1}\right)^{N}} \leq \frac{u}{\left(\bar{M} L^{k-1}\right)^{N-\frac{1}{k-1}}} \leq u
$$

and inductively, for all $i=0, \ldots, k-1$,

$$
\left|z_{i}\right| \leq \frac{L^{i} u}{\left(\bar{M} L^{k-1}\right)^{N}} \leq \frac{u}{\left(\bar{M} L^{k-1}\right)^{N-\frac{i}{k-1}}} \leq u .
$$

So

$$
\begin{aligned}
& \left|z_{k}\right| \leq \mathcal{L}\left(\alpha, l, \xi_{k}, u\right)\left|z_{k-1}\right| \\
\leq & \frac{\bar{M} L^{k-1} u}{\left(\bar{M} L^{k-1}\right)^{N}}=\frac{u}{\left(\bar{M} L^{k-1}\right)^{N-1}} \leq u .
\end{aligned}
$$

Similarly, for any $j<N$,

$$
\begin{aligned}
\left|z_{j k}\right| & \leq \mathcal{L}\left(\alpha, l, \xi_{j k}, u\right)\left|z_{j k-1}\right| \\
& \leq \mathcal{L}\left(\alpha, l, \xi_{j k}, u\right) L^{k-1}\left|z_{j(k-1)}\right| \\
& \leq\left(L^{k-1}\right)^{j} \prod_{i=1}^{j} \mathcal{L}\left(\alpha, l, \xi_{i k}, u\right)\left|z_{0}\right| \\
& <\left(\bar{M} L^{k-1}\right)^{j}\left|z_{0}\right|<\frac{u}{\left(\bar{M} L^{k-1}\right)^{N-j}} \leq u .
\end{aligned}
$$

Denoting $\bar{\lambda}:=[\lambda-(k-1) \ln L] / 2$, and applying (A.1), we get, on $\Omega_{\gamma}$,

$$
\begin{aligned}
\left|z_{k N}\right| & \leq L^{(k-1) N}\left|z_{0}\right| \prod_{i=1}^{N} \mathcal{L}\left(\alpha, l, \xi_{i k}, u\right) \\
& \leq\left|z_{0}\right| e^{((k-1) N) \ln L} e^{-\frac{[\lambda+(k-1) \ln L] N}{2}}=e^{-\bar{\lambda} N}\left|z_{0}\right|<u,
\end{aligned}
$$

and then, for each $j=1,2, \ldots, k-1$,

$$
\begin{aligned}
\left|z_{k N+j}\right| & <L^{j}\left|z_{k N}\right|<e^{-\bar{\lambda} N} L^{j}\left|z_{0}\right| \\
& <e^{-\bar{\lambda} N} \frac{u}{\bar{M}^{N} L^{(k-1) N-j}}<u .
\end{aligned}
$$

Similarly, for any $n=k m+j$, where $j=1,2, \ldots, k-1$, $m>N$, we get, for $\omega \in \Omega_{\gamma}$,

$$
\begin{aligned}
\left|z_{n}(\omega)\right| & <L^{j}\left|z_{k m}(\omega)\right|<e^{-\bar{\lambda} k m} L^{j}\left|z_{0}\right| \leq e^{-\bar{\lambda}(n-j)} L^{k}\left|z_{0}\right| \\
& <e^{-\bar{\lambda}(n-j)} \frac{u}{\bar{M}^{N} L^{(k-1) N-k}}<u e^{-\bar{\lambda}(n-j)}<u,
\end{aligned}
$$


which implies that $\lim _{n \rightarrow \infty} z_{n}(\omega)=0$ when $\omega \in \Omega_{\gamma}$ and concludes the proof of Case (b).

Remark. If $M L^{k-1}>1$ but $M<1$, we may use much bigger initial interval than it was suggested in the proof of Theorem I.4 assuming $\delta_{0} \leq \frac{u}{M L^{k-1}}$ for part (a) and $\delta_{0} \leq \frac{u}{\left(M L^{k-1}\right)^{N}}$ for part (b) of the proof. However, in this case we need to change model (7) slightly, considering instead

$$
z_{n+1}=\left\{\begin{aligned}
G\left(z_{n}, \alpha, l, \xi_{n+1}\right), & n=k(s-1), \quad s \in \mathbb{N} \\
g\left(z_{n}\right), & \text { otherwise, } \quad z_{0} \in \mathbb{R} .
\end{aligned}\right.
$$

In A.3 , the application of stochastic control starts from $n=0$ rather than $n=s-1$. Note that in both cases, the solution $z_{n}$ remains in $[-u, u]$ for all $n \in \mathbb{N}$. The proof differs only in the estimation of the first $k$ (respectively $N k$ ) iterations.

\section{Proof of Lemma}

Let $i=1$, other cases are similar. Note that $\prod_{s=j}^{d} \max \left\{L_{s}, 1\right\} \geq 1$ for $j=0, \ldots, d$, and

$$
K_{1}=K_{d+1}=f\left(K_{d}\right)=f^{d-j}\left(K_{d-j+1}\right)=f^{d}\left(K_{1}\right) .
$$

For $\left|x-K_{1}\right| \leq u_{0}\left(\prod_{s=1}^{d} \max \left\{L_{s}, 1\right\}\right)^{-1}$, we have

$$
\begin{aligned}
\left|f(x)-K_{2}\right| & \leq \max \left\{L_{1}, 1\right\}\left|x-K_{1}\right| \\
& \leq u_{0}\left(\prod_{s=2}^{d} \max \left\{L_{s}, 1\right\}\right)^{-1} \leq u_{0}
\end{aligned}
$$

and, inductively, for each $j=0, \ldots, d-1$,

$$
\begin{aligned}
& \quad\left|f^{d-j}(x)-K_{d-j+1}\right| \leq \prod_{s=1}^{d-j} \max \left\{L_{s}, 1\right\}\left|x-K_{1}\right| \\
& \leq u_{0}\left(\prod_{s=d-j+1}^{d} \max \left\{L_{s}, 1\right\}\right)^{-1} \leq u_{0},
\end{aligned}
$$

which, for $j=0$, implies (14).

\section{Proof of Lemma}

Let $u(d)$ be defined as in (16). Under Assumption \.9. we have, for $x \in\left[K_{i}-u(d), K_{i}+u(d)\right]$,

$$
\begin{aligned}
\left|f(x)-K_{i+1}\right| & \leq\left|\mathcal{A}_{i}\left(x-K_{i}\right)\right|+\left|\phi_{i}(x)\right| \\
& \leq\left[\left|\mathcal{A}_{i}\right|+\psi_{i}(u)\right]\left|x-K_{i}\right|
\end{aligned}
$$

so (12) holds for $L_{i}:=\left|\mathcal{A}_{i}\right|+\psi_{i}(u)$. Acting as in the proof of Lemma $\amalg .8$ we obtain that, for $j=1, \ldots, d$,

$$
\begin{aligned}
& \left|f^{j}(x)-K_{j+1}\right| \leq \prod_{i=1}^{j}\left[\left|\mathcal{A}_{i}\right|+\psi_{i}(u)\right]\left|x-K_{1}\right| \\
\leq & \frac{u}{\prod_{i=j+1}^{d} \max \left\{\left|\mathcal{A}_{i}\right|+\psi_{i}(u), 1\right\}} \leq u,
\end{aligned}
$$

where, notationally, $\prod_{i}^{j} \cdot=1$ for any $i>j$. Now apply (15) recursively, for $\left|x-K_{1}\right|<u(d)$,

$$
\begin{aligned}
& f^{d}(x)-K_{1} \\
= & \mathcal{A}_{d}\left(\mathcal{A}_{d-1}\left(f^{d-2}(x)-K_{d-1}\right)\right. \\
& \left.+\phi_{d-1}\left(f^{d-2}(x)\right)\right)+\phi_{d}\left(f^{d-1}(x)\right) \\
& \vdots \\
= & \left(\prod_{i=1}^{d} \mathcal{A}_{i}\right)\left(x-K_{1}\right)+\sum_{j=1}^{d-1}\left(\prod_{i=j+1}^{d} \mathcal{A}_{i}\right) \phi_{j}\left(f^{j-1}(x)\right) \\
& +\phi_{d}\left(f^{d-1}(x)\right),
\end{aligned}
$$

where $f^{0}(x):=x$. Define

$$
\bar{\phi}(x):=\sum_{j=1}^{d}\left(\prod_{s=j+1}^{d} \mathcal{A}_{s}\right) \phi_{j}\left(f^{j-1}(x)\right) .
$$

Acting as above we get, for $j=1, \ldots, d$,

$$
\begin{aligned}
& \left|\phi_{j}\left(f^{j-1}(x)\right)\right| \\
\leq & \psi_{j}\left(\left|f^{j-1}(x)-K_{j}\right|\right)\left|f^{j-1}(x)-K_{j}\right| \\
\leq & \psi_{j}\left(\left|f^{j-1}(x)-K_{j}\right|\right)\left[\left|\mathcal{A}_{j-1}\right|+\psi_{j-1}(u)\right] \\
& \times\left|f^{j-2}(x)-K_{j-1}\right| \\
& \leq \psi_{j}\left(\left|f^{j-1}(x)-K_{j}\right|\right) \prod_{i=1}^{j-1}\left[\left|\mathcal{A}_{i}\right|+\psi_{i}(u)\right]\left|x-K_{1}\right| .
\end{aligned}
$$

So we can set

$$
\begin{aligned}
\bar{\psi}(x):=b & \sum_{j=1}^{d}\left(\prod_{s=j+1}^{d} \mathcal{A}_{s}\right) \psi_{j}\left(\left|f^{j-1}(x)-K_{j}\right|\right) \\
& \times \prod_{i=1}^{j-1}\left(\left|\mathcal{A}_{i}\right|+\psi_{i}(u)\right)
\end{aligned}
$$

which completes the proof of (17).

\section{Proof of Lemma}

An application of Theorem II.4 implies the existence of $s_{0}$ and $\Omega_{\gamma} \subset \Omega$ with $\mathbb{P}\left(\Omega_{\gamma}\right) \geq 1-\gamma$ such that, for sufficiently small $\delta>0, s \geq s_{0}, \omega \in \Omega_{\gamma}$

$$
\left|x_{s m d}(\omega)-K_{1}\right|<u_{0} L^{-m}(d)|1-\alpha+l|^{-m+1} .
$$


We need to show that, for each $s \geq s_{0}, j=q d+\bar{j}$, $\bar{j}=1, \ldots, d-2$, and $q \leq m-1$, we have $\mid x_{s m d+j}(\omega)-$ $K_{\bar{j}+1} \mid \leq u_{0}$ when $\omega \in \Omega_{\gamma}$, which allows us to apply (12) on each step.

Indeed, $\left|x_{s m d+1}-K_{2}\right|=\left|f\left(x_{s m d}\right)-f\left(K_{1}\right)\right| \leq$ $L_{1}\left|x_{s m d}-K_{1}\right| \leq u_{0}$. Reasoning inductively, we have for each $j=1, \ldots, d-1$ and $\omega \in \Omega_{\gamma}$,

$$
\begin{aligned}
& \left|x_{s m d+j}(\omega)-K_{j+1}\right|=\left|f\left(x_{s m d+j-1}(\omega)\right)-f\left(K_{j}\right)\right| \\
& \leq \prod_{i=1}^{j} L_{i}\left|x_{s m d}(\omega)-K_{1}\right| \leq u_{0}, \\
& \left|x_{s m d+d}(\omega)-K_{d+1}\right| \\
& \leq|1-\alpha+l| L(d)\left|x_{s m d}(\omega)-K_{1}\right| \leq u_{0},
\end{aligned}
$$

and for $q=d+1, \ldots, 2 d-1$ and again $\omega \in \Omega_{\gamma}$,

$$
\begin{aligned}
& \left|x_{s m d+q}(\omega)-K_{q-d+1}\right| \\
\leq & L_{q-d}\left|x_{s m d+q-1}(\omega)-K_{q-d}\right| \\
\leq & \cdots \leq \prod_{\theta=1}^{q-d} L_{\theta}\left|x_{s m d+q-d}(\omega)-K_{1}\right| \\
\leq & L(d)|1-\alpha+l| \prod_{\theta=1}^{q-d} L_{\theta}\left|x_{s m d}(\omega)-K_{1}\right| \leq u_{0} .
\end{aligned}
$$

Similarly, for $j=q d+\bar{j}, \bar{j}=1, \ldots, d-2, q \leq m-1$, and for $\omega \in \Omega_{\gamma}$,

$$
\begin{aligned}
& \left|x_{s m d+j}(\omega)-K_{\bar{j}+1}\right| \\
\leq & L_{\bar{j}}\left|x_{s m d+j-1}(\omega)-K_{\bar{j}}\right| \\
\leq & \cdots \leq|1-\alpha+l|^{q} \prod_{\theta=1}^{\bar{j}} L_{\theta} L^{q}(d)\left|x_{s m d}(\omega)-K_{1}\right| \\
\leq & |1-\alpha+l|^{q} L^{q+1}(d)\left|x_{s m d}(\omega)-K_{1}\right| \\
\leq & |1-\alpha+l|^{m-1} L^{m}(d)\left|x_{s m d}-K_{1}\right| \leq u_{0} .
\end{aligned}
$$

\section{Proof of Theorem}

Consider (19) with arbitrary $x_{0}$ and assume that (23) holds. Then, for any $\gamma \in(0,1)$, there exists $\Omega_{\gamma} \subset \Omega$ with $\mathbb{P}\left(\Omega_{\gamma}\right)>1-\gamma$ and $N \in \mathbb{N}$ such that for $\bar{\lambda}=\frac{1}{2}(\lambda-k \ln \bar{L})$, we have on $\Omega_{\gamma}$, for $n=t k+j, j=1,2, \ldots, k-1$, and $t=\lfloor n / k\rfloor>N$, where $\lfloor q\rfloor$ is an integer part of $q \in[0, \infty)$,

$$
\begin{aligned}
& \left(L^{k}\left|1-\alpha-l \xi_{n}\right|\right)^{t} \leq e^{-\bar{\lambda} t}, \\
& \left|x_{n}-K\right| \leq L^{t k+j}\left|1-\alpha-l \xi_{n}\right|^{t}\left|x_{0}-K\right| \\
\leq & e^{-\bar{\lambda} t} L^{k}\left|x_{0}-K\right|,
\end{aligned}
$$

which tends to zero as $n \rightarrow \infty$ (so that $k \rightarrow \infty$ ). Here note that condition (23) holds with $L$ substituted by the global constant $\bar{L}$, which implies that $\lambda-k \ln \bar{L}>0$, and then $\bar{\lambda}>0$.
Analogously, consider (26) with arbitrary $x_{0}$, and assume that (29) holds. Then condition (29) is satisfied with $L(d)$ substituted by the global constant $\bar{L}(d)$, which implies that $\lambda-m \ln \bar{L}(d)>0$. Applying Lemma I.13 we get, for $\bar{\lambda}=(\lambda-m \ln L(d)) / 2, n=t m d+j$, $t=\lfloor u /(m d)\rfloor>N, j=q d+\bar{j}, \bar{j}=0,1, \ldots, d-1$, $q=0,1, \ldots, m-1$,

$$
\begin{aligned}
& \left(L^{m}(d)\left|1-\alpha-l \xi_{n}\right|\right)^{t} \leq e^{-\bar{\lambda} t}, \\
& \left|x_{n}-K_{\bar{j}+1}\right|=\left|x_{t m d+j}-K_{\bar{j}+1}\right| \\
\leq & |1-\alpha+l|^{m-1} L^{m}(d)\left|x_{t m d}-K_{1}\right| \\
\leq & e^{-\bar{\lambda} t}|1-\alpha+l|^{m-1} L^{m}(d)\left|x_{0}-K\right| \rightarrow 0, \text { as } m \rightarrow \infty,
\end{aligned}
$$

and therefore $n \rightarrow \infty$.

If $M L^{k}<1$ (respectively, $M L(d)<1$ ), each of limits above holds for all $\omega \in \Omega$.

\section{Proof of Theorem 11.18}

Let $G(z, \alpha, l, v)$ be defined as in (31) and denote, for simplicity, $G:=G(z, \alpha, l, v)$. Let $z \in\left[-u_{0}, 0\right]$ and $G>0$, then

$$
\begin{aligned}
|G| & =(1-\alpha-l v) g(z)+(\alpha+l v) z<(1-\alpha-l v) g(z) \\
& =(1-\alpha-l v) g(z) \leq(1-\alpha-l v) L|z| .
\end{aligned}
$$

If $G \leq 0$, we have

$$
|G|=-(1-\alpha-l v) g(z)-(\alpha+l v) z<(\alpha+l v)|z| .
$$

Now, let $z \in\left[0, u_{0}\right]$ and $G>0$, then

$$
|G|=(1-\alpha-l v) g(z)+(\alpha+l v) z<(\alpha+l v)|z| .
$$

If $G \leq 0$,

$$
\begin{aligned}
|G| & =-(1-\alpha-l v) g(z)-(\alpha+l v) z \\
& <(1-\alpha-l v)|g(z)| \leq(1-\alpha-l v) L|z| .
\end{aligned}
$$

So (8) holds for $\mathcal{L}(\alpha, l, v, u)=\max _{|v| \leq 1}\{(1-\alpha-l v) L, \alpha+$ $l v\}$, and (9) is satisfied for $M=\max \{(1-\alpha+l) L, \alpha+l\}$. Condition (10) then takes the form

$$
\min \{-\mathbb{E} \ln |1+\alpha|,-\mathbb{E} \ln |1-\alpha-l \xi| L\} \geq(k-1) \ln L .
$$

A direct application of Theorem $\amalg .4$ proves Part (ii).

The condition $M L^{k-1}<1$ takes the form $\max \{(1-$ $\alpha+l) L, \alpha+l\} L^{k-1}<1$. In the case where $k=1$ and all $L>1$, this implies the conditions listed in (33) in Part (i). In the case where $k>1$ and for all $L$ satisfying $1<L^{k}<L+1$, this implies the conditions in (34) in Part (i). Another application of Theorem $\amalg .4$ concludes the proof. 


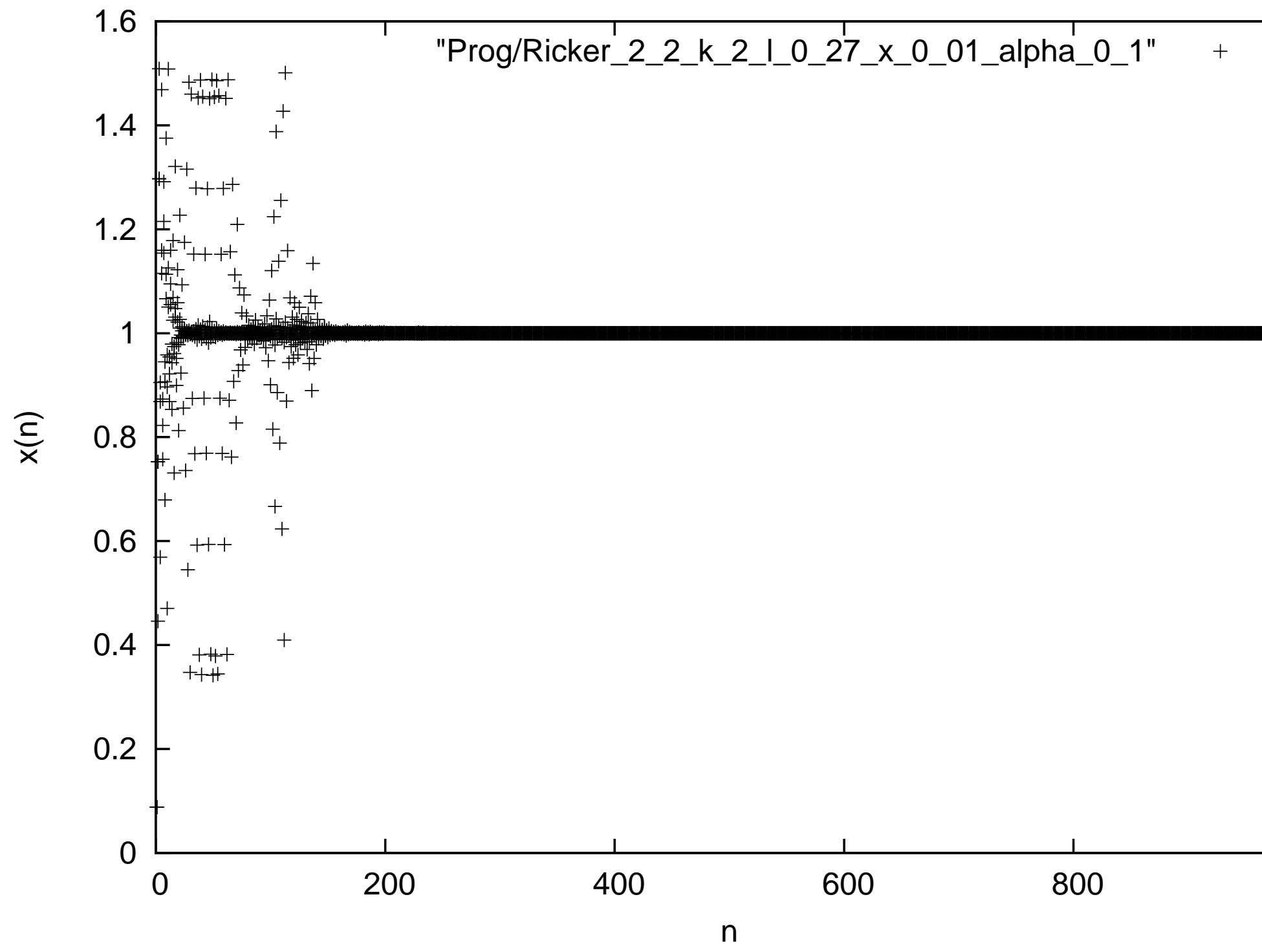




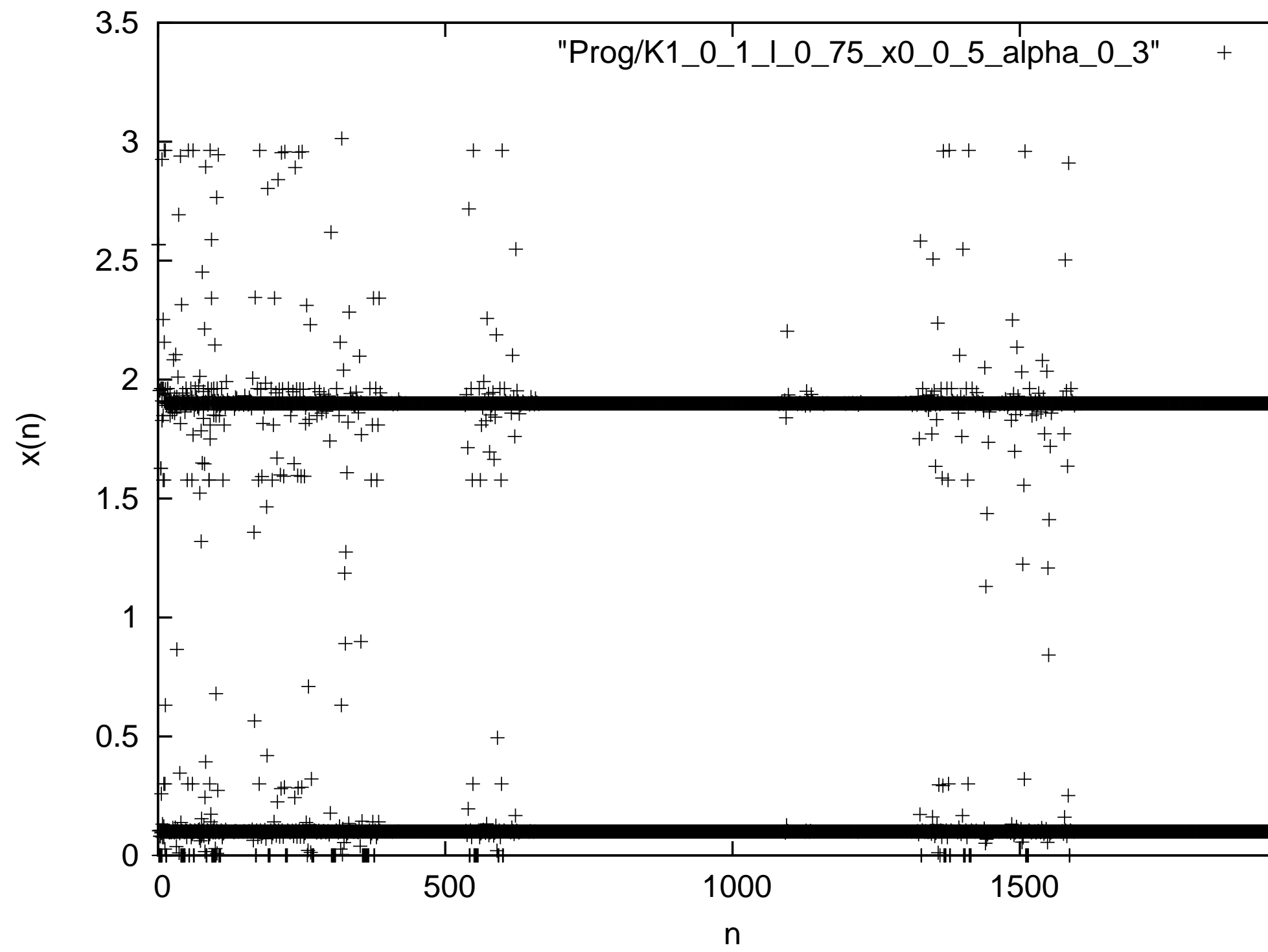




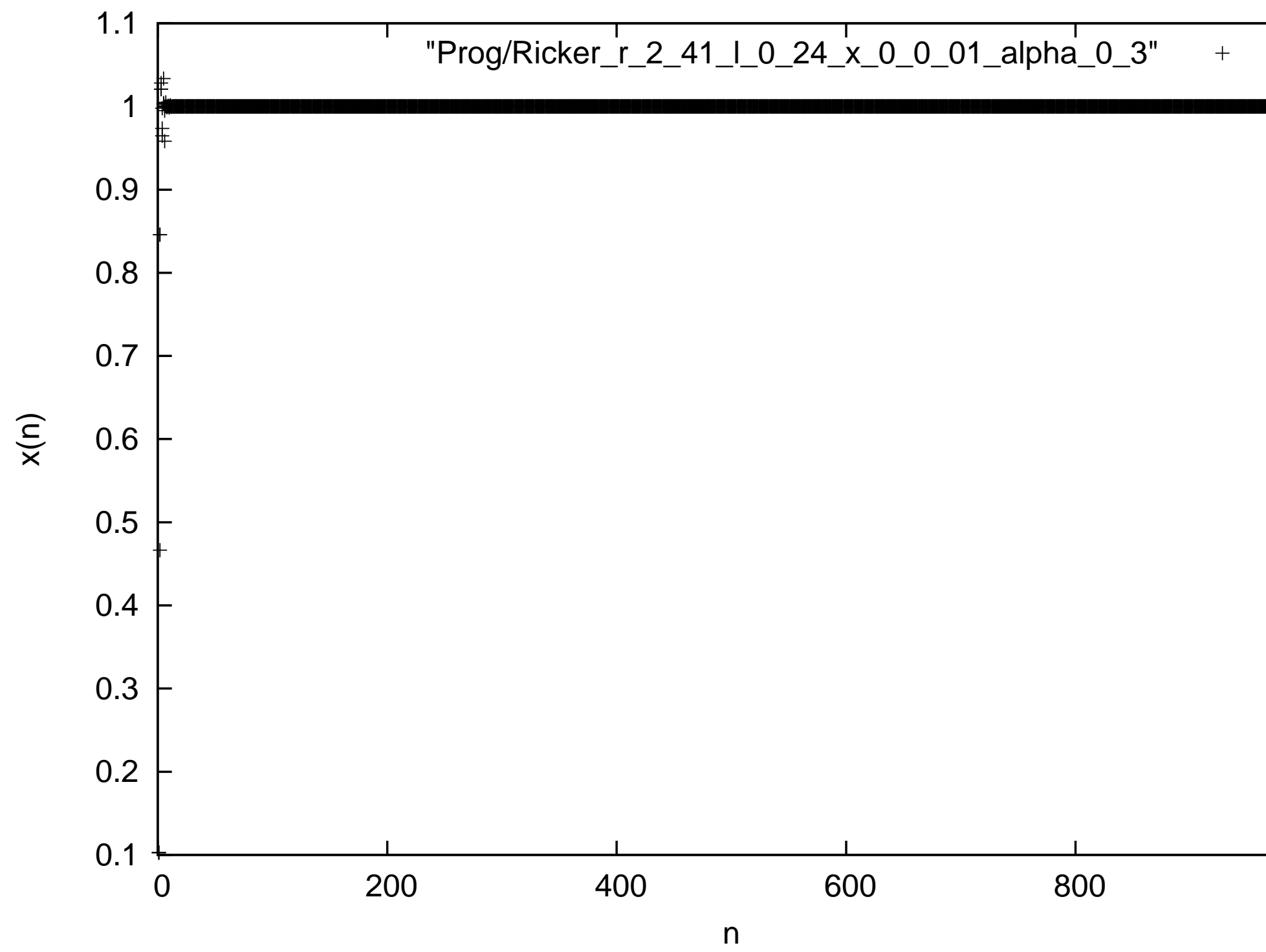




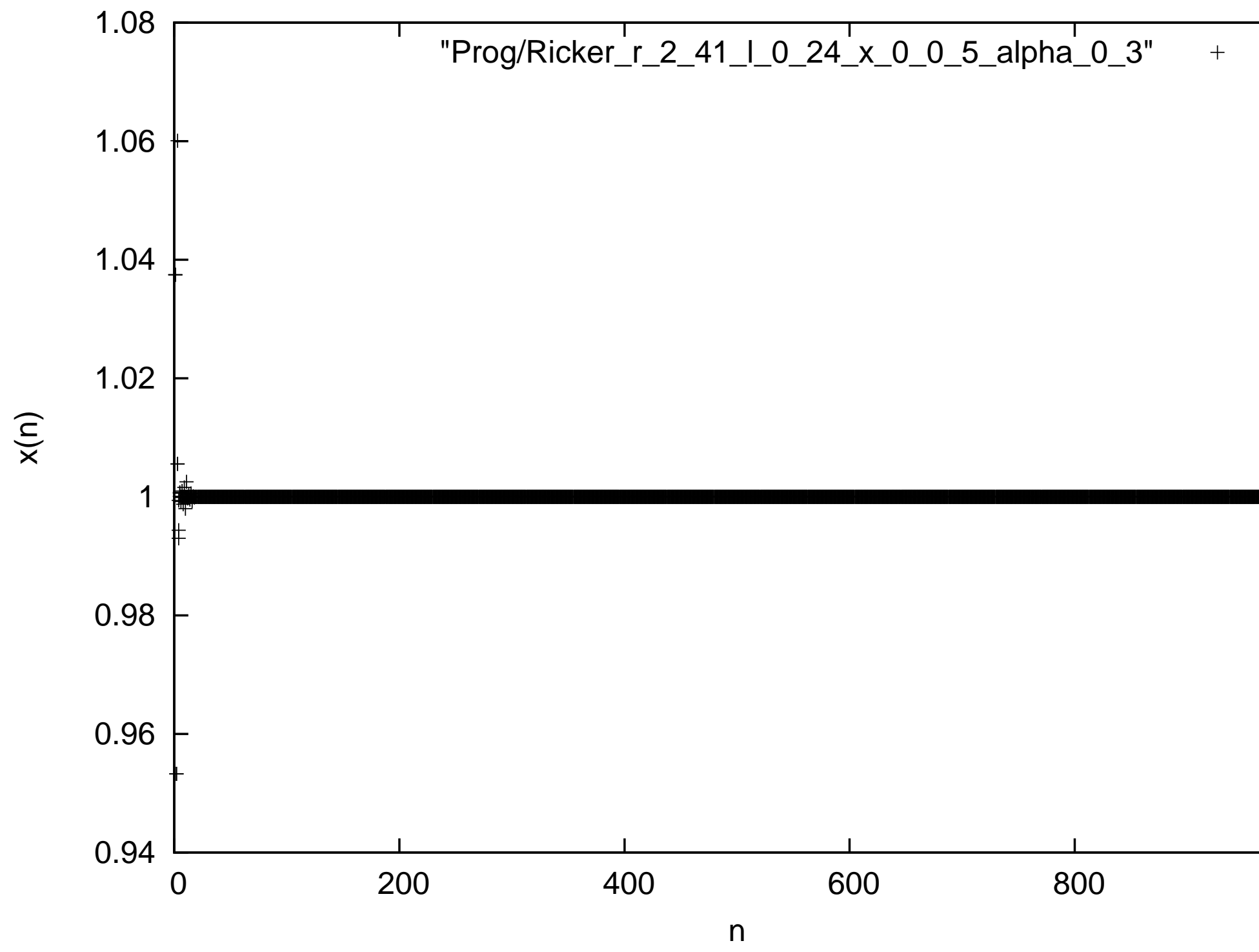




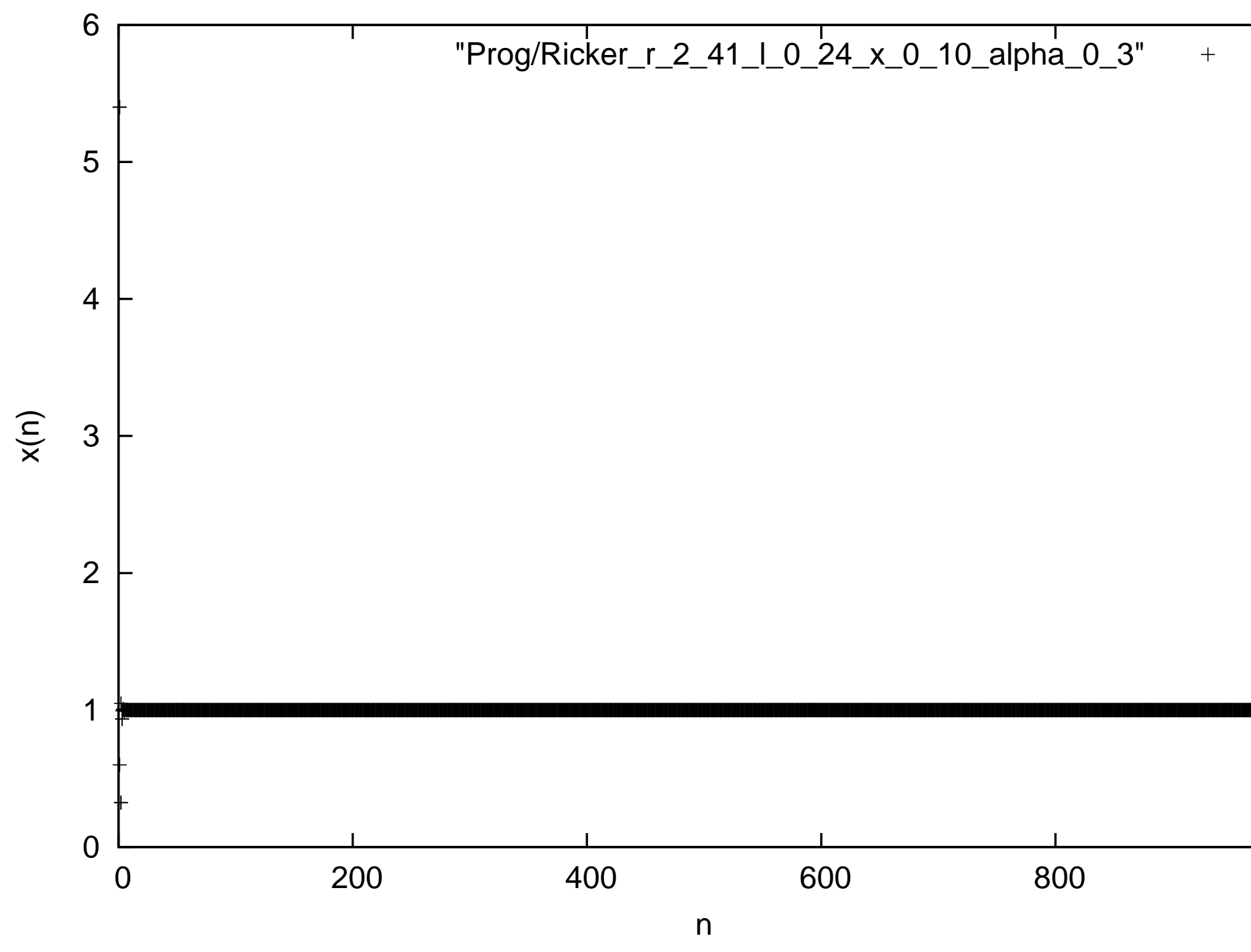




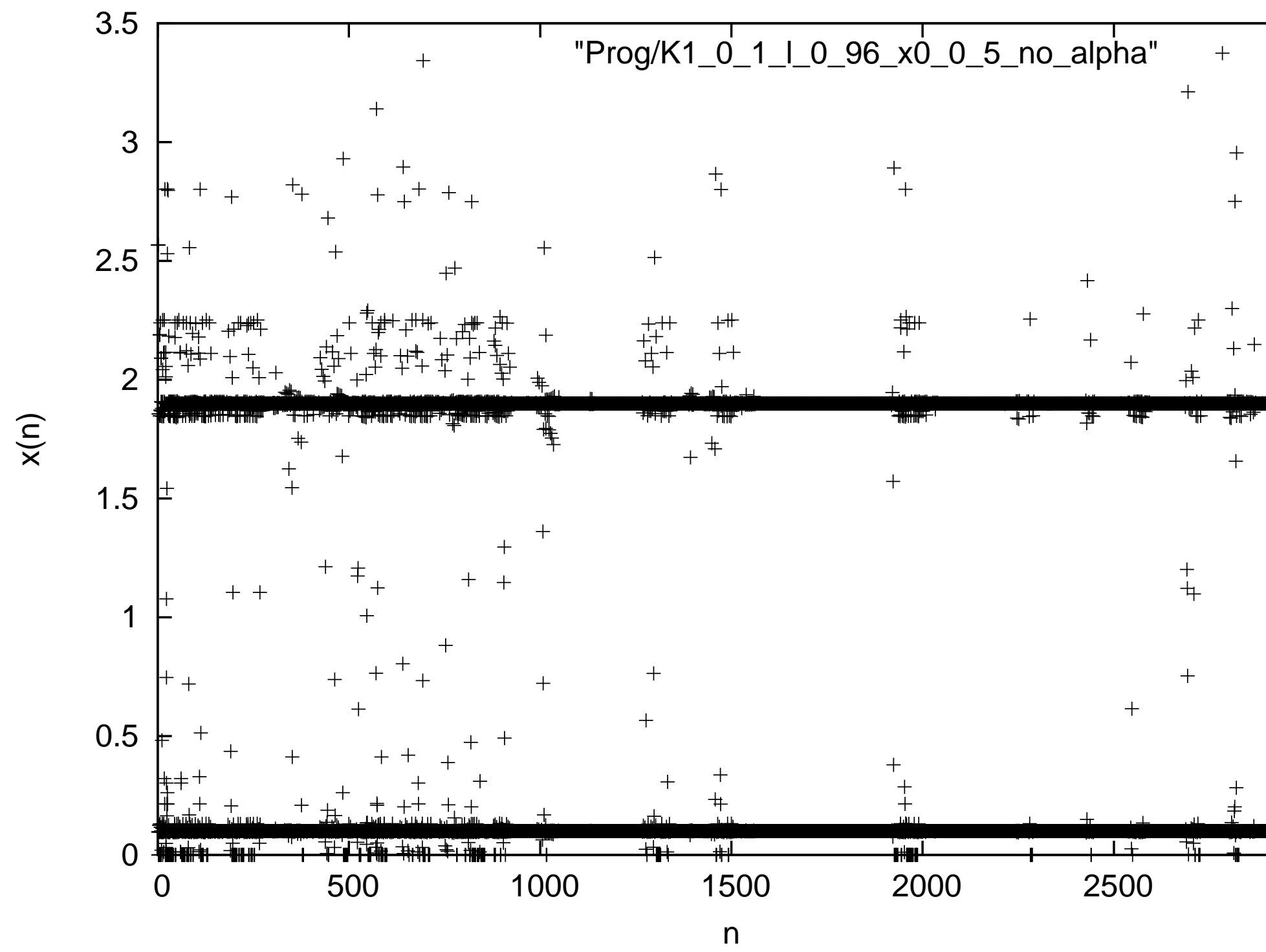




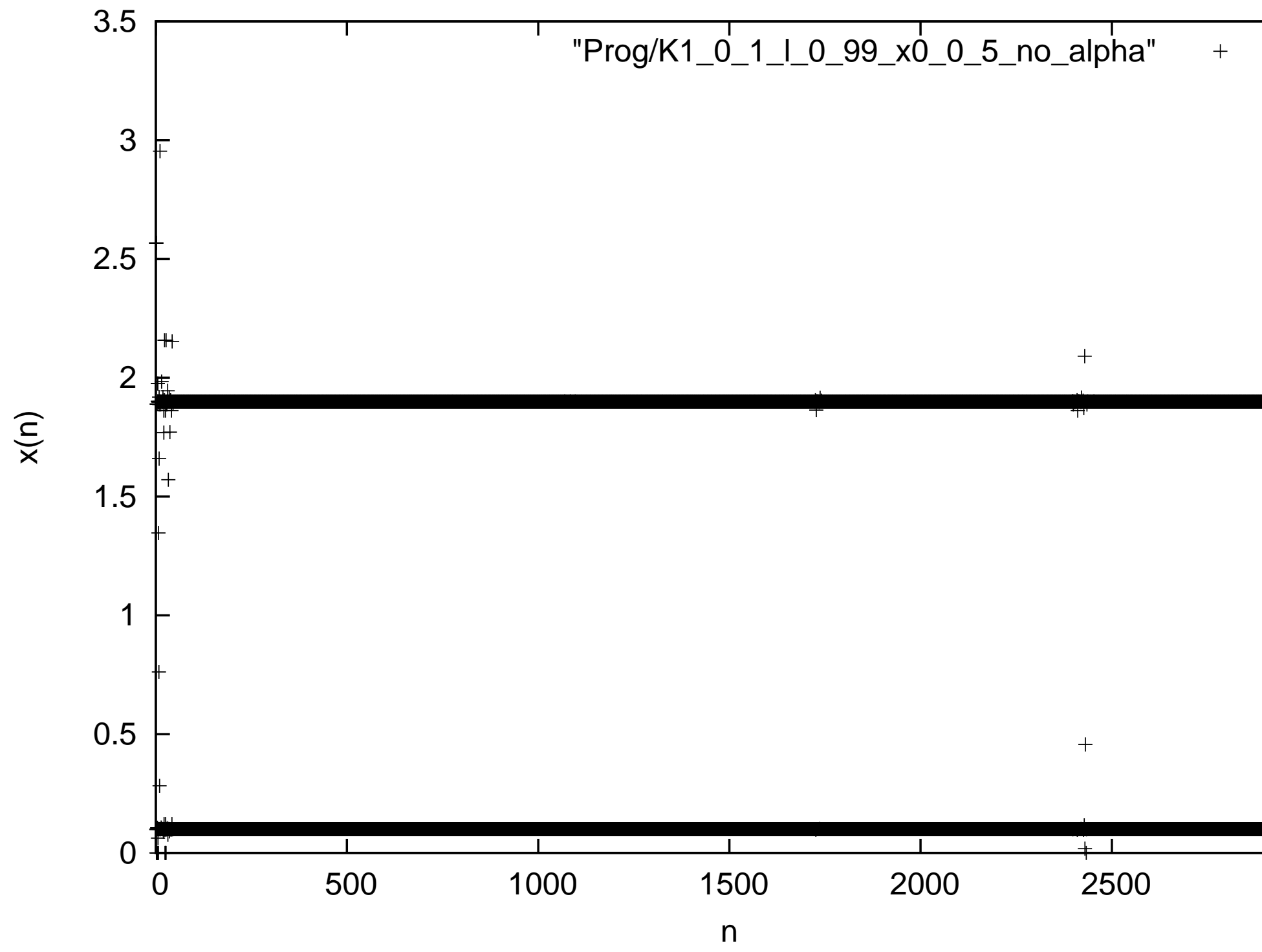




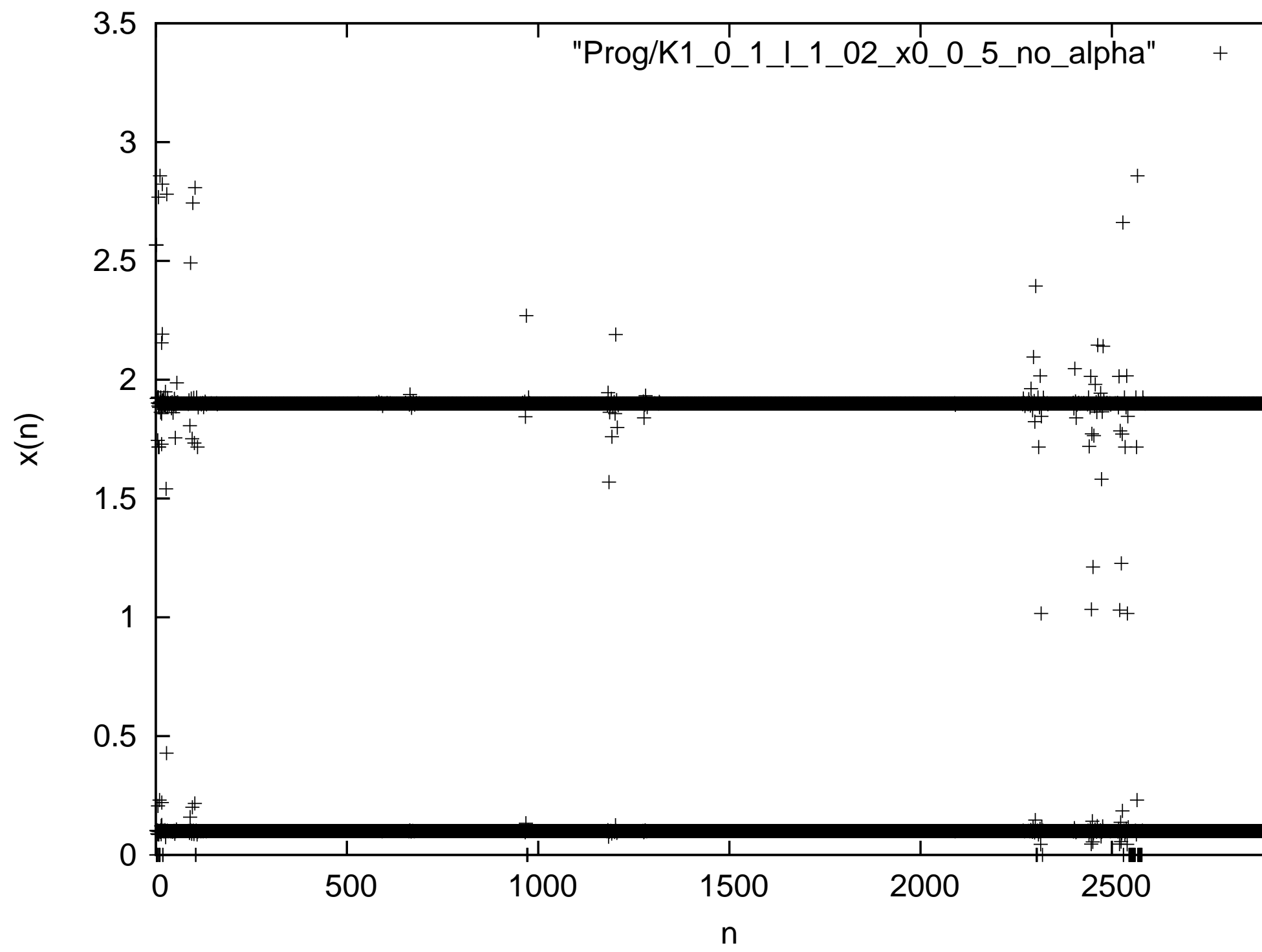




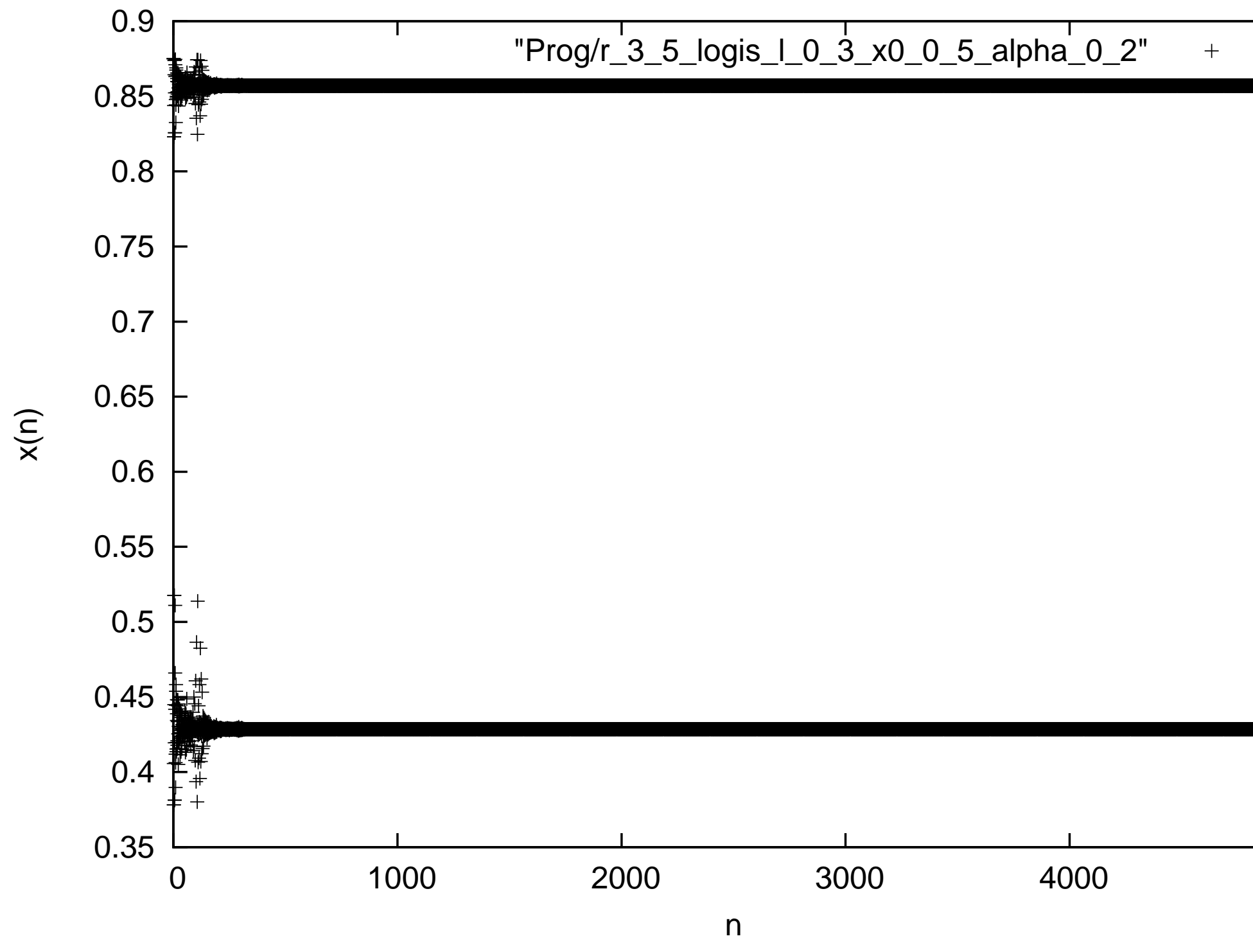




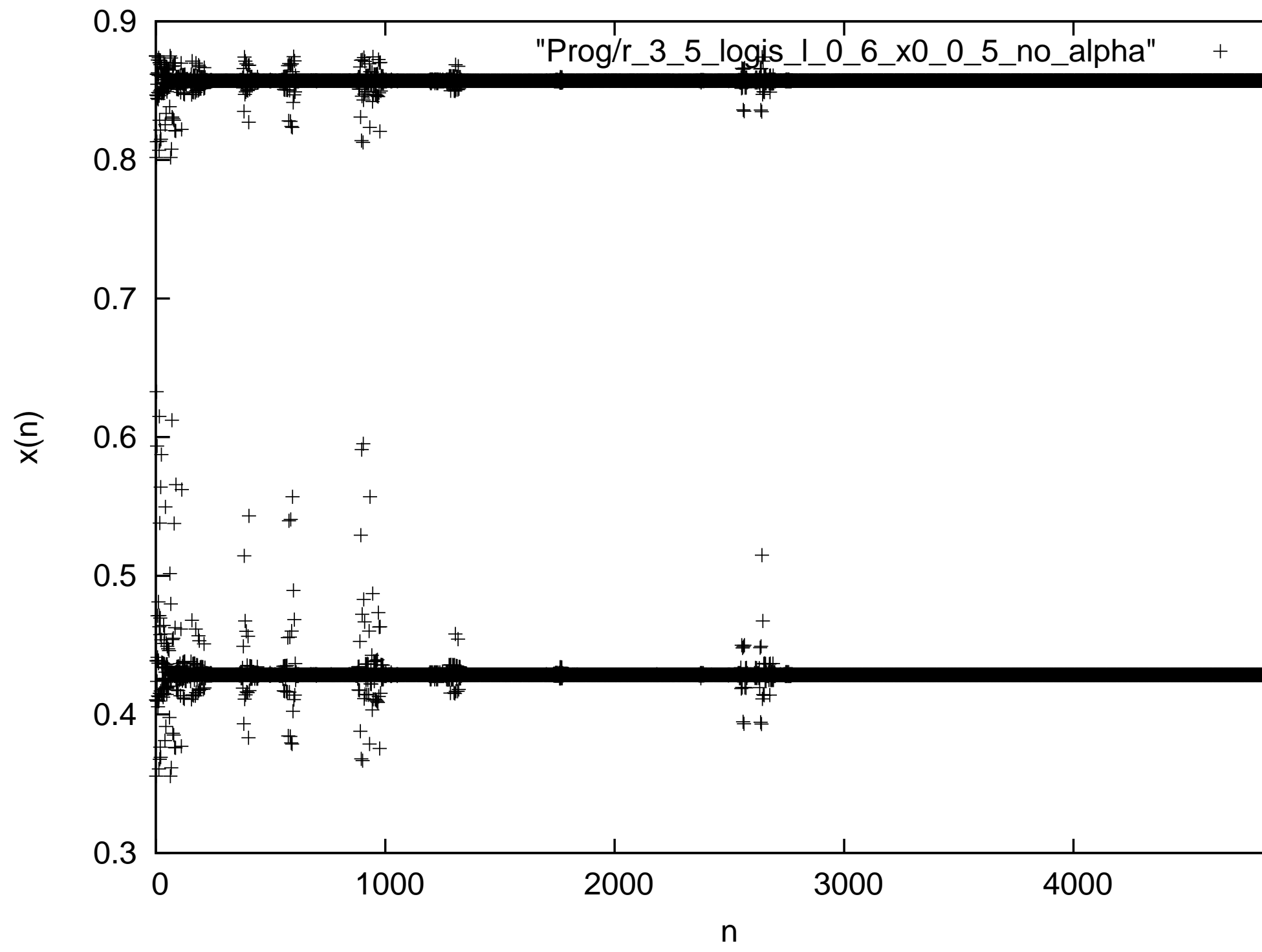




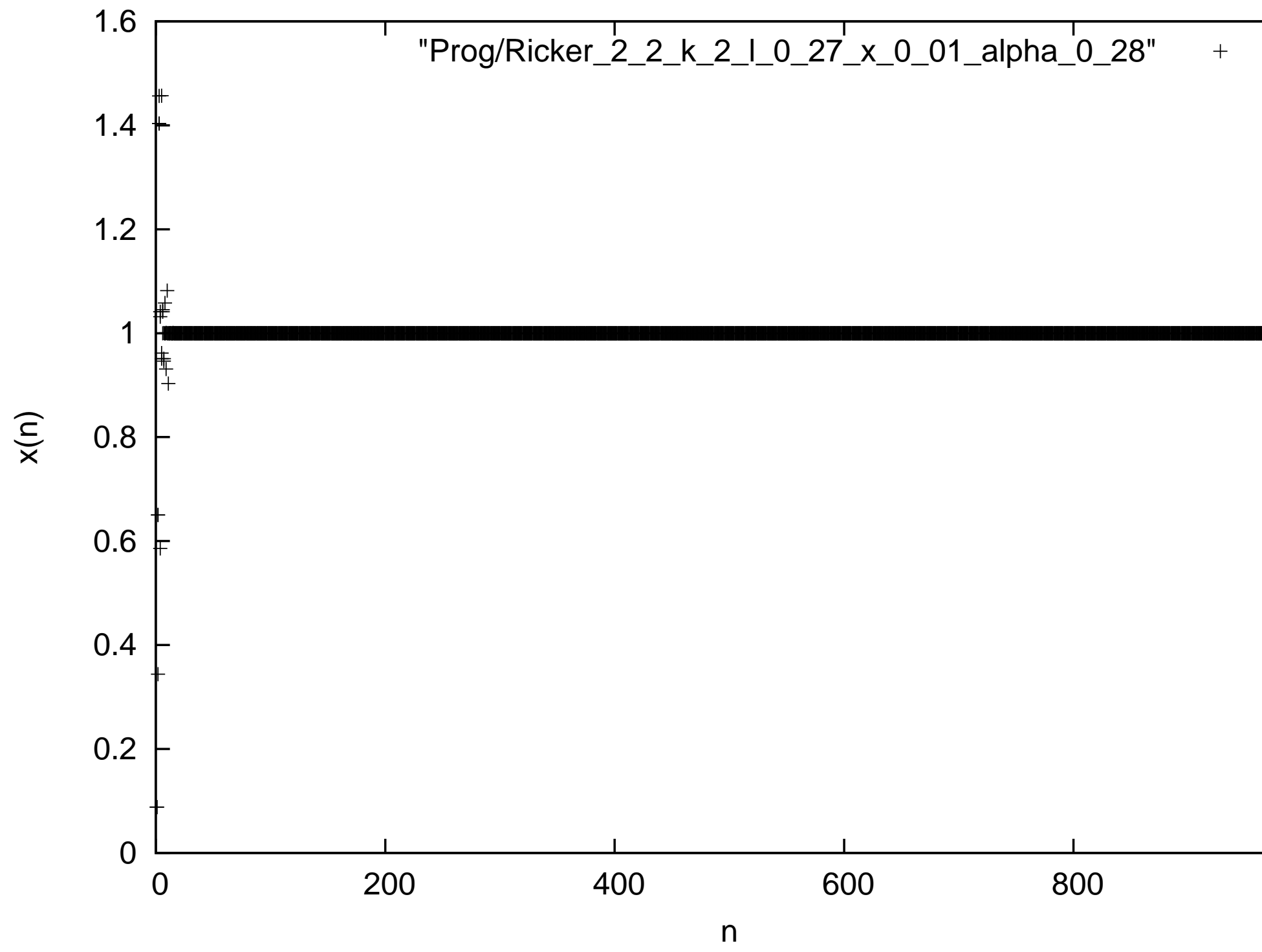

